\title{
Growth competition between columnar dendritic grains - The role of microstructural length scales
}

\author{
Elaheh Dorari ${ }^{1}$, Kaihua $\mathrm{Ji}^{1}$, Gildas Guillemot ${ }^{2}$, Charles-André Gandin ${ }^{2}$ and Alain Karma ${ }^{1}$ \\ ${ }^{1}$ Department of Physics and Center for Inter-disciplinary Research on Complex Systems, \\ Northeastern University, Boston, MA 02115, USA \\ ${ }^{2}$ MINES ParisTech, PSL Research University, CEMEF UMR CNRS 7635, CS10207, \\ 06904 Sophia Antipolis, France
}

\begin{abstract}
The growth competition between columnar dendritic grains is investigated by both phasefield (PF) and cellular automaton (CA) models in a growth regime where the primary dendrite spacing is much larger than the solutal diffusion length. This growth regime favors the formation of highly branched hierarchical dendritic microstructures prevalent in castings. Previous PF and CA studies have shed light on the complex relationship between GB orientation selection and GB bi-crystallography. They showed that, in the CA model, the orientation is governed by the favorably oriented grain (FOG) criterion and the geometrical limit (GL) in the limit of large and small cell size, respectively. The present study focuses on exploring how to quantitatively bridge length scales between PF simulations that resolve the whole solid-liquid interface dynamics and the CA model that resolves the dynamics of the grain envelope under a certain set of assumptions. For this purpose, we study grain boundary (GB) orientation selection as a function of the imposed temperature gradient $G$ under the frozen-temperature approximation, which allows us to vary $G$ dependent microstructural length scales with a fixed GB bi-crystallography. PF simulations reveal
\end{abstract}


the existence of a transition from FOG to GL dominance with decreasing $G$ at non-degenerate bicrystallography. Simulations further reveal that this transition can be quantitatively reproduced by the CA model with a choice of the cell size that corresponds, in PF simulations, to the active secondary dendrite arm spacing of the favorably oriented grain preceding the tertiary branching event that gives birth to a new stable primary dendrite. PF simulations are also used to obtain a detailed quantitative characterization of the dynamics of the grain envelope and its internal length scales, thereby providing a quantitative test of the inherent approximations of the existing CA approach and paving the way for its future development.

Keywords: Phase-field modeling, Cellular automaton modeling, Solidification, Growth competition, Grain boundary 


\section{Introduction}

During alloy solidification in material processing such as casting and additive manufacturing, competitive growth occurs between grains with different preferred crystal directions and hence grain boundaries of various orientations form. Microstructural features, especially at the GB locations determine the material properties. Highly branched dendrites can form at the GB locations, with microstructural measures such as dendrite tip radius, primary and secondary arm spacing, the distance behind the tip at which tertiary branching events occur, leading to a new stable primary dendrite as well as competitive crystal growth and GB orientation angles. Unlike a stand-alone dendritic microstructure or intra-grain dendritic features, which can be characterized with the help of theoretical models [1,2], characterization of the GB microstructural features is a much more complex task. While recent numerical simulations have clarified some mechanisms of GB orientation selection (e.g., Refs. [3-6]), a comprehensive picture of this complex phenomenon accounting for the role of microstructural length scales and the influence of cooling conditions is still lacking.

In attempts to understand the mechanism of the GB orientation selection, Walton and Chalmers [7] proposed a selection model for competitive grain growth. They suggested that the grain with the lowest misorientation $|\alpha|$ with respect to the thermal gradient direction cannot be overgrown. Based on their model, an interpretation, namely the Favorably Oriented Grain criterion, was developed assuming that the GB follows the growth direction of the lowest angle $|\alpha|$ grain also known as the most favorably-oriented grain [8]. Considering a setup schematically illustrated in Fig. 1, where the orientation of a GB (located by orange dashed lines in Fig. 1a and b) is defined as $\theta$, and $\alpha_{1}$ and $\alpha_{2}$ are the crystal angles of the grains located, respectively, on the top and bottom hand side of the GB, the FOG criterion predicts the orientation selection map 
depicted in Fig. 2a. This interpretation of Walton and Chalmers's model was initially accepted, but later experimental observations [9-12] challenged the FOG criterion.

Another GB selection criterion derived by Pineau et al. [8], termed the geometrical limit, is established by assuming that the boundary of the mushy zone is a continuous interface and is defined mathematically by considering the limit where the growth length of all dendrite branches is considered as infinitely small. In this limit, branching at a diverging GB occurs equally from both grains, which leads to a grain boundary orientation given by the bisecting line of the primary growth directions of columnar dendrites in the two grains. In the case of converging primary directions, two scenarios are defined assuming branching takes place solely from the top hand side grain or the bottom hand side grain, whichever has the smallest absolute crystal angle, i.e., the most advanced grain profile along the solidification direction. Assuming a steady growth regime, the profile of the mushy zone boundary defined by a bi-crystal is stable during growth and translates with constant velocity along the GB. Fig. $2 b$ represents the GB orientation map corresponding to the GL criterion.

Large three-dimensional (3D) PF simulations of columnar dendritic grains could be achieved to extract an overall behavior of the grain density evolutions as a function of the temperature gradient [13-15]. However, in-depth numerical studies of growth competition, branching formation, and evolution at the GB are needed to explain the experimental observations [12,15-17]. Recent phase-field simulations have been developed to elucidate complex mechanisms of growth competition at the grain boundaries, e.g., unusual overgrowth at the converging GBs [3-6], [18-20] mainly observed in cellular or near dendritic regime and branching at the diverging GBs $[4,6]$. The PF method enables quantitative modeling of dendritic growth at the scale of the solid-liquid interface and sidebranching competition. Simulation of alloy 
solidification at experimentally relevant length and time scales was made possible through the introduction of the thin-interface limit and the anti-trapping current [21]. In our recent study [22], we introduced an improved finite-difference implementation of the PF model of directional alloy solidification in which spatial derivative terms appearing in the evolution equations for the solute field (the divergences of the chemical potential gradient and the anti-trapping current) and the phase-field (standard Laplacian term) remain rotationally invariant up to second order in the grid spacing. This improved finite difference scheme avoids grid-induced anisotropy and makes it possible to accurately resolve both the growth orientation and the tip operating state of misoriented dendrites, which is controlled by the small anisotropy of the excess free-energy of the solid-liquid interface in a velocity regime where dendritic microstructures are well developed.

In previously published articles, quantitative phase-field simulations were performed to investigate the macroscopic grain boundary orientation selection in bi-crystalline directional solidification of a binary succinonitrile $0.4 \mathrm{wt} . \%$ acetone with pulling velocity $25 \mu \mathrm{m} \mathrm{s}^{-1}[4,6]$. The temperature gradient $1000-30000 \mathrm{~K} \mathrm{~m}^{-1}$ used in the study of Tourret and Karma [4], covered the formation of weakly to fully branched, i.e., near cellular to dendritic arrays with the order of magnitude of the primary arm spacing from sidebranching of the misoriented grain $O\left(10^{0}-10^{2}\right) \mu \mathrm{m}$. Smaller arm spacings are related to higher temperature gradients under which near cellular arrays form, while the new primary branching at the GB emerges directly from a unique curved sidebranch. At smaller temperature gradients, distinct secondary and tertiary branches form with increased primary spacing. The study of Tourret and Karma [4] was performed in two dimensions (2D) and only considered the situation with one misoriented dendritic grain competing with a well-oriented grain growing in the temperature gradient direction. In terms of 
characterization of the microstructural length scales, ref. [4] was limited to the primary arm spacings from sidebranching of the misoriented grain.

From Tourret et al. [6], at fixed temperature gradient $3000 \mathrm{~K} \mathrm{~m}^{-1}$, the entire orientation range for the two grains in 2D was mapped, and deviations of the GB orientation from the FOG criterion were identified by Pineau et al. [8]. A revised-Favorably Oriented Grain criterion (revFOG) was proposed to interpret the results of the PF simulations. For the aforementioned alloy composition and cooling condition, the rev-FOG criterion was introduced to capture deviations from FOG at: a) diverging GBs with $\alpha_{1} \times \alpha_{2}<0$, which exhibit the highest lateral mobility during growth and results in noisy fluctuations of their average trajectory around $\theta \approx 0^{\circ} ; \mathrm{b}$ ) converging GBs with degenerate growth and $\alpha_{1} \times \alpha_{2}>0$, in the presence of one or both highly-misoriented grains in the vicinity of $|\alpha|=45^{\circ}$, which yields a transition $\theta \rightarrow 0^{\circ}$; c) the converging GBs with $\left|\alpha_{1}+\alpha_{2}\right| \leq 5^{\circ}$ which show a transition between $\theta \rightarrow \alpha_{1}$ and $\theta \rightarrow \alpha_{2}$ due to the occurrence of the so-called "unusual overgrowth" of favorably-oriented dendrites. Fig. 2c represents the GB orientation map corresponding to the rev-FOG criterion. This study did not characterize the GB microstructural length scales or transitions between FOG and GL. 


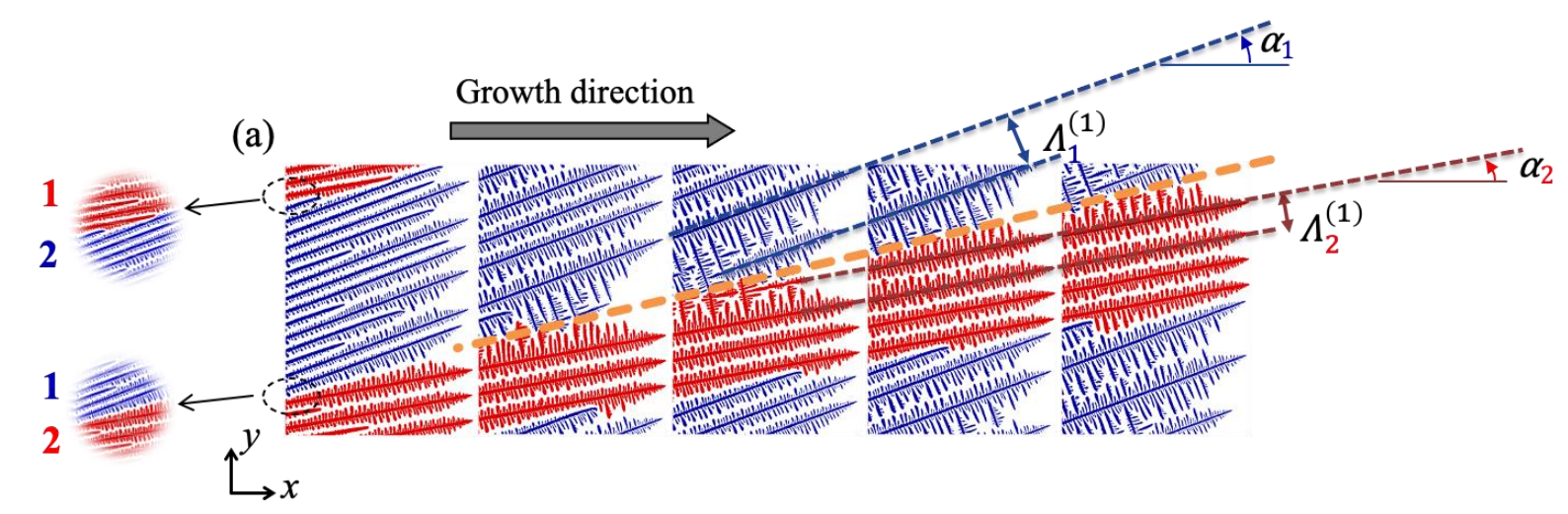

(b)
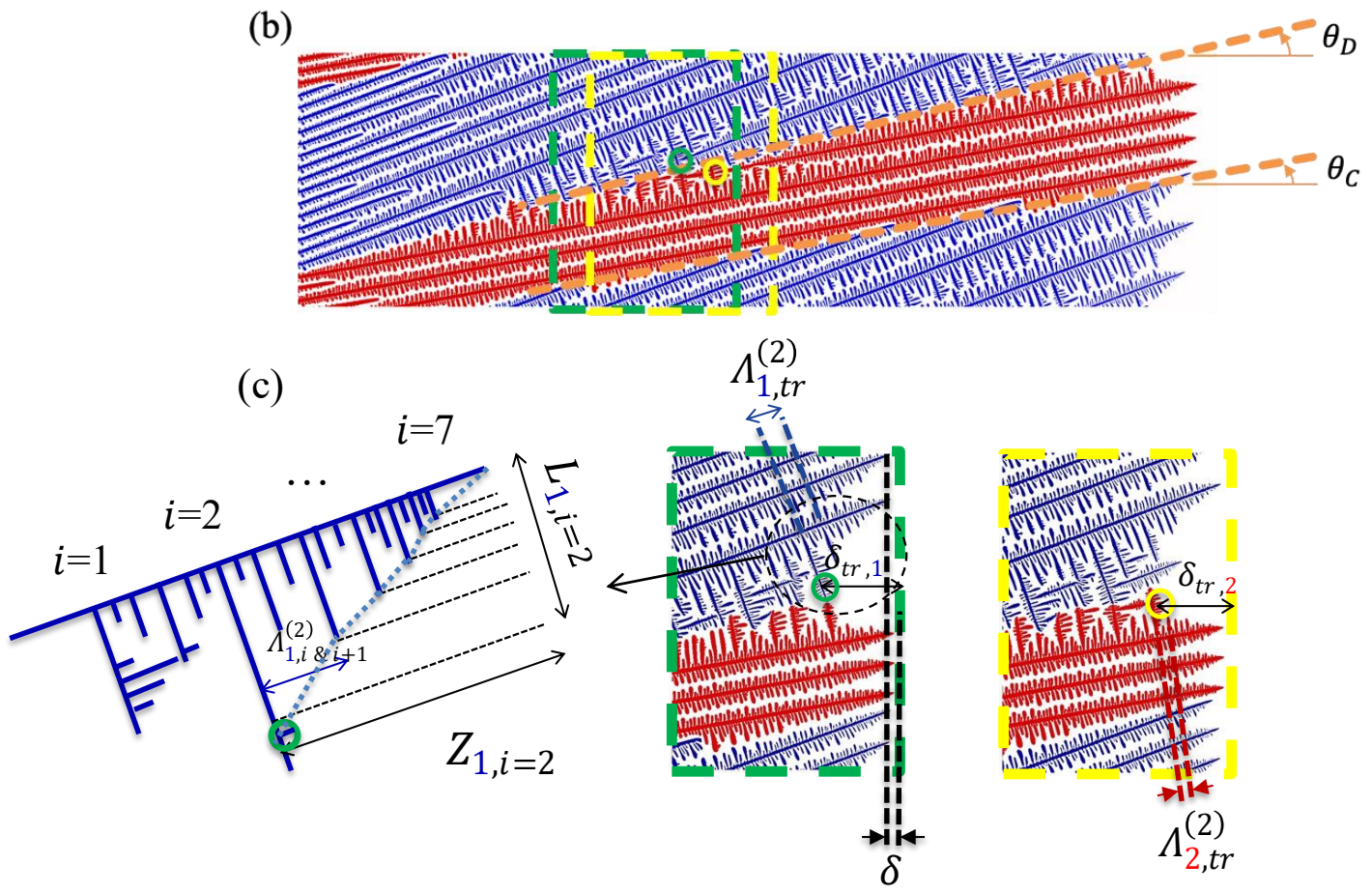

Fig. 1. Microstructural length scales and orientations of the bi-crystal deduced from PF simulations of the growth history in directional solidification with a temperature gradient $(G)$ in the horizontal $+x$ direction and periodic boundaries in the $y$-direction. (a) Primary dendritic spacing, $\Lambda^{(1)}$, resulting from branching events within grain $1, \Lambda_{1}^{(1)}$, and grain $2, \Lambda_{2}^{(1)}$, at the diverging GB shown by the orange dashed line. $\alpha_{1}$ and $\alpha_{2}$ are the crystal angle of the grains located, respectively, on the top and bottom of the diverging GB. (b) Linear fits to the diverging and converging GBs with orientation angles $\theta_{D}$ and $\theta_{C}$, respectively, as well as the frames associated with the emergence of stable tertiary branches at the diverging GB region on grain 1 (green frame) and grain 2 (yellow frame). (c) Snapshot frames of the emergence of the stable tertiary branches on grain 1 and grain 2 at the diverging GB. Active secondary arm spacing preceding the start of a stable tertiary branch 
on grain 1 and 2 at locations circled by green and yellow is shown by $\Lambda_{1, t r}^{(2)}$ and $\Lambda_{2, t r}^{(2)}$, respectively. The depth from the most advanced tip at which a stable tertiary branch is going to start on grain 1 and 2 within the GB is shown by $\delta_{t r, 1}$ and $\delta_{t r, 2}$, respectively. Spacing $\Lambda_{1 / 2, i \& i+1}^{(2)}$, length $L_{1 / 2, i}$ and distance from the tip of the trunk $Z_{1 / 2, i}$ are measured for seven consecutive secondary branches in diverging GB region, as schematized for grain 1 only. $\delta$ ( $\delta_{S t}$ in steady state) is the distance between the most advanced tips of the two grains.

(a)

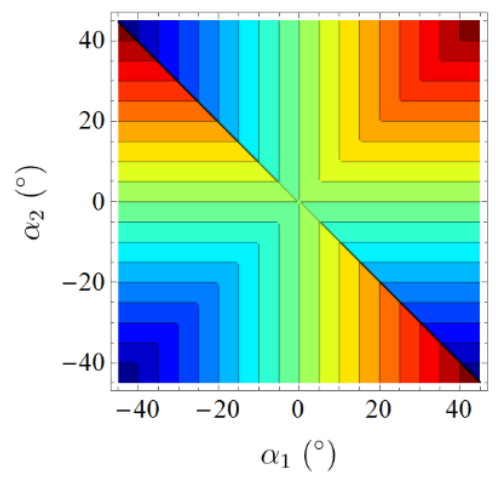

(b)

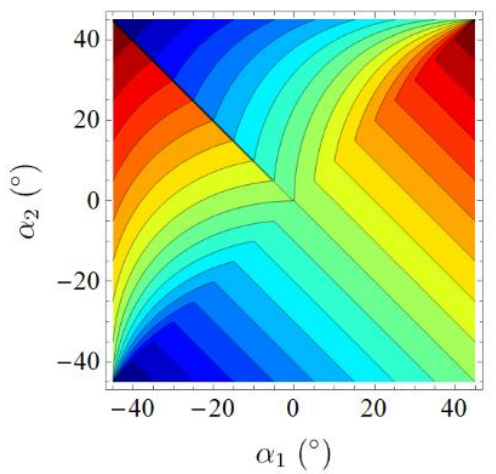

(c)

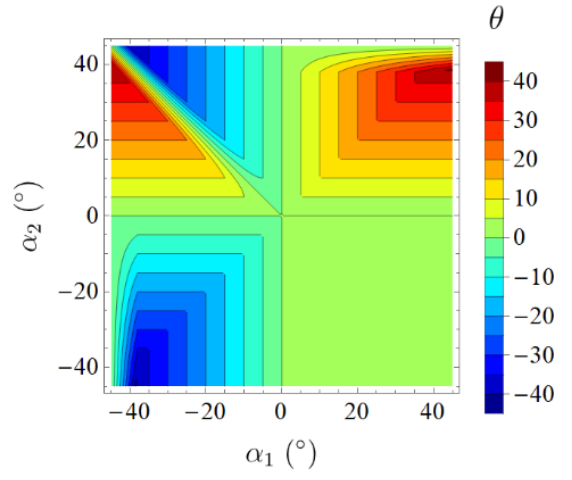

Fig. 2. Grain boundary orientation selection maps corresponding to the (a) FOG, (b) GL, and (c) rev-FOG criteria [6] shown as color maps of the GB angle, $\theta$, versus the crystal angles $\alpha_{1}$ and $\alpha_{2}$ of the grains located, on the top and bottom of the bi-crystal shown in Fig. 1, respectively.

Operating on a larger scale, cellular automaton modeling aims at simulating the grain envelope evolution rather than the complex underlying dendritic microstructures [23]. This simplification enables computational times several orders of magnitudes lower than PF. It was introduced to reach large dimensions of the simulation domains while coupling the nucleation and growth of the solidifying dendritic grains with solutions for energy, momentum, and solute mass conservations using the finite element (FE) method $[14,24,25]$. The so-called CAFE model could be used to study growth competition among columnar dendritic grains, leading to crystallographic textured columnar zones, as well as between grain morphologies, leading to columnar-to-equiaxed transitions $[15,26]$. While this could be achieved for 3D columnar grain structures, only a global comparison with experimental data was achieved for grain selection taking place among a large 
population of columnar grains [15]. The methodology provides a valuable tool for the prediction of texture formation in various solidification processes $[15,24]$, but requires additional and more in-depth analyses of its behavior at grain boundaries.

Pineau et al. [8] conducted 2D comparisons of CA versus PF simulations for the prediction of GB orientation under the same condition as Tourret et al. [6]. Comparisons were developed to investigate cell size effect on GB angle evolution in growth competition between two grains during directional solidification. The distance between the front of the two grains presented as $\delta$ in Fig. 1c, at steady state (denoted by $\delta_{S t}$ ), under a given temperature gradient was considered as the threshold length to discuss the growth mechanism. For a cell size, $l_{C A}$, lower than $\delta_{S t}$, the distance between simulated dendrite arms is small enough to mimic branching mechanisms in various $\langle 10\rangle$ directions for the growing cells located in the GB domain. This simulation condition is related to the hypothesis made for the construction of the GL criterion and, hence CA prediction of GB angle converges to GL, i.e., $\theta \simeq \theta_{G L}$. On the other hand, if the cell size, $l_{C A}$, is larger than $\delta_{S t}$, the branching events are inhibited in the GB domain. The grain with the lower misorientation grows ahead at a lower undercooling and imposes the GB orientation leading to $\theta \simeq \theta_{F O G}$. For both conditions $l_{C A} \ll \delta_{S t}$ and $l_{C A} \gg \delta_{S t}$, convergence is observed toward these two analytical solutions as intrinsic limits of the CA model. Pineau et al. [8] empirically found that by selecting an intermediate cell size of the order of the maximum step between the two competing grains $\left(l_{C A} \simeq\right.$ $\delta_{S t}$ at $\left.\left(\alpha_{1}=0^{\circ}, \alpha_{2}=45^{\circ}\right)\right)$, excellent agreement could be found with the rev-FOG criterion derived from PF simulations over a wide range of grain orientations. This suggests that the cell size in the CA model may be related to a characteristic length associated with the branching mechanism developed between two grains of fixed crystal orientation at their common boundary, which can generally depend on the history of the temperature gradient and isotherm velocity. 
However, Ref. [8] did not pinpoint this characteristic length scale and determine if it can be meaningfully extracted from PF simulations.

The goal of this paper is to pinpoint how this characteristic length, which is associated with branch competition in the GB region, can be extracted from the PF simulations to enable the CA model make quantitative predictions of GB orientation selection on the grain scale. To this end, we perform a comprehensive study of microstructural characteristics and GB orientation selection during growth competition between columnar fully-developed dendritic grains in a wellestablished dendritic regime where the primary dendrite spacing is much larger than the solutal diffusion length as depicted in Fig. 1. Since we do not know a priori which microstructural length scale is most relevant to determine the cell size in the CA model, we consider several length scales displayed in Fig. 1a-c together with the bi-crystal and GB angles, which include primary spacings and secondary branch spacings prior to tertiary branching events.

Primary spacings and bi-crystallography. We denote the primary dendritic spacing $\Lambda^{(1)}$ resulting from branching events within grain $1, \Lambda_{1}^{(1)}$, and grain $2, \Lambda_{2}^{(1)}$ at the diverging GB region shown by the orange dashed line; $\alpha_{1}$ and $\alpha_{2}$ are the crystal angle of the grains located, respectively, on the top and bottom hand side of the diverging GB sketched assuming $\left|\alpha_{1}\right|>\left|\alpha_{2}\right|$. $\alpha_{1}$ and $\alpha_{2}$ are interchangeable when moving from one GB to the neighbor GB. All angles are defined counterclockwise with respect to the $+x$ direction so that, here, both $\alpha_{1}$ and $\alpha_{2}>0$. Fig. 1b exhibits the growth history and linear fits to the diverging and converging GBs with orientation angles $\theta_{D}$ and $\theta_{C}$, respectively, as well as the frames associated with the emergence of stable tertiary branches at the diverging GB region on grain 1 (green frame) and grain 2 (yellow frame). Secondary branch spacing prior to tertiary branching events. Fig. 1c shows the snapshot frames of the emergence of the stable tertiary branches on grain 1 and grain 2 at the diverging GB 
region. Note that the stable tertiary branching cannot be determined a priori based on any snapshot of the microstructure. As schematized, many tertiaries form from secondary branches but only one for each grain may eventually become stable. For a new primary, the time evolution is rewound to find its origin as a tertiary close to the tip of an active secondary branch. From the retrieved snapshots, all the following listed quantities are measured. The microstructural length scales depicted in Fig. 1c include active secondary arm spacing preceding the start of a stable tertiary branch on grain 1 and 2 at locations circled by green and yellow, respectively shown by $\Lambda_{1, t r}^{(2)}$ and $\Lambda_{2, t r}^{(2)}$. The depth from the most advanced tip at which a stable tertiary branch is going to start on grain 1 and 2 within the GB region is shown by $\delta_{t r, 1}$ and $\delta_{t r, 2}$, respectively. Spacing $\Lambda_{1 / 2, i \& i+1}^{(2)}$, length $L_{1 / 2, i}$, and distance from the tip of the trunk $Z_{1 / 2, i}$ for seven consecutive secondary branches in the diverging GB region at the instances that a stable tertiary branching starts on grain 1 or 2 (i.e., 1/2) are characterized; $i=2$ is related to the active secondary arm on which a stable tertiary branch emerges, and active tips ahead are indexed $i>2$. A sidebranch is considered active if it is longer than any other sidebranch that has originated closer to the tip of the parent branch. $i=1$ is related to a passive secondary before tertiary branching and within the grain history. The grain envelope is constructed as lines connecting the tip of the primary dendrite and active sidebranches (dashed line in Fig. 1c). $\delta$ is the distance between the most advanced tips of the two grains. All the aforementioned parameters are measured at a steady state defined by constant $\delta=\delta_{S t}$. Throughout the text, when needed, the overline symbol is used to represent the average value of these parameters. In Fig. 1, for clarity, subscripts 1 and 2 that represent grain 1 and grain 2 are colored the same as their corresponding grain, respectively blue and red. 
Previous experiments have been carried out under growth conditions leading to the GL dominance regimes $[12,16]$. Existing quantitative PF simulations $[4,6]$, however, have been conducted under growth conditions where the GB orientation selection falls in the FOG or revFOG regime. Lack of simulations at GL dominance regime may partially be due to the significant increase in computational cost associated with increased length and time scales, and perhaps more importantly, due to the complex task of resolving the growth orientation and the tip operating state of the misoriented dendrites in that regime. In this study, simulations are performed for varying imposed temperature gradients $G$ in so called frozen-temperature approximation [4] under which GB orientation selection transitions from FOG to GL dominance regime. For this purpose, we use the rotationally invariant finite difference implementation of the PF model [22] since it is ideally suited to investigate, quantitatively, the grain competition in a velocity regime where dendritic microstructures are well developed, as shown in Fig. 1, free of spurious grid anisotropy effects.

Observations of the relationship between processing condition and microstructural length scales in directional solidification have been limited to intra-grain primary and secondary dendrite arm spacings. Inter-grain microstructural characterization of grain competition is still lacking, and the study by Tourret and Karma [4] was limited to the primary arm spacing. Most importantly, the role of the microstructural length scales in grain competition has not been studied. Even though the previous study [4] showed the failure of the assumption that the elimination rate of the misoriented grain always increases with increased distance between the most advanced tips of the two grains, $\delta_{S t}$, this naive assumption is still commonly made. In this study, the role of the microstructural length scales in transition from FOG to GL dominance is systematically studied. For this purpose, PF simulations are carried out to obtain a detailed quantitative characterization of the dynamics of the grain envelope and the internal $G$-dependent microstructural length scales 
shown in Fig. 1 (primary spacing $\Lambda^{(1)}$, secondary arm spacing $\Lambda^{(2)}$, the depth of emergence of a stable tertiary branch, $\delta_{t r}$, from the most advanced tip, as well as $\delta_{S t}$ ).

Furthermore, the present study explores how to quantitatively bridge length scales between PF simulations that resolve the whole solid-liquid interface dynamics and the CA model that resolves the dynamics of the grain envelope under a certain set of assumptions. The previous study by Pineau et al. [8] in bridging CA and PF was limited to the rev-FOG regime and solely explored the length scale, $\delta_{S t}$. In the current study, we use the quantitative measurements of the grain envelope, microstructural length scales, and GB orientation selection obtained from PF simulations and described in Fig.1, in order to identify the relevant CA cell size for bridging CA and PF prediction of the GB orientation. The bridging length scale for a varying temperature gradient that covers the FOG to GL dominance regime is explored and the appropriate selection is identified. The performance of the CA with its current inherent approximations in the prediction of the GB orientation and grain envelopes is tested, which paves the path for its future development.

\section{Methods}

\subsection{Phase-field simulations}

We used a quantitative PF formulation with thin-interface limit and corrective anti-trapping current for directional solidification of a dilute binary alloy with diffusive solute transport in the liquid and no diffusion in solid [21]. The thin-interface asymptotics analysis allows using a width of the diffuse interface $W$ much larger than the capillarity length $d_{0}$ described as [27]:

$$
d_{0}=\frac{\Gamma}{|m| c_{0}\left(\frac{1}{k}-1\right)}
$$

where $\Gamma$ is the Gibbs-Thomson coefficient of the solid-liquid interface, $c_{0}$ is the nominal solute concentration of the alloy, and $k$ is the interface solute partition coefficient. The corrective anti- 
trapping current was used in PF equations to counterbalance the spurious solute trapping associated with the jump of chemical potential across the interface [28]. The second-order rotationally invariant finite-difference scheme for the spatial derivative terms of the solute and phase-field equations developed in our recent work [22] was used to accurately resolve both the growth orientation and the dendrite tip operating state of the misoriented grains at larger interface thickness $W$, larger grid size $\Delta x$, and smaller anisotropy $\varepsilon$ than previously feasible. We implemented the reformulated equations of the PF model as in Ref. [29] using a non-linear preconditioning of the phase-field parameter $\varphi$ as $\varphi(x, y, t)=\tanh \{\psi(x, y, t) / \sqrt{2}\}$ in order to enhance the numerical stability of the equations for larger grid spacings. The equations were solved using finite differences and an explicit time scheme. The convergence of the model with respect to the diffuse interface thickness and grid size was validated in order to determine appropriate values of $\frac{W}{d_{0}}$ and $\frac{\Delta x}{W}$, as reported in Table 1 .

In order to imitate thermal noise which is physically known to be the origin of dendritic sidebranching [30], a stochastic force is added to the evolution of the $\psi$ field as explained in detail in Ref. [29]. The model was implemented for parallel processing on Graphics Processing Units (GPU) using the Compute Unified Device Architecture (CUDA) programming language developed by Nvidia. The isotropic finite difference scheme used in the current study is computationally more costly (close to 3 times [22]) than the common PF discretization in previous studies [6,29] but still achievable on a single GPU. Details on the equations and the second-order isotropic discretization can be found in [29] and [22], respectively.

We aim at characterizing the growth competition behavior, i.e., GB orientation, microstructural length scales, and grain envelope in bi-crystalline directional solidification. A 
succinonitrile $-1.3 \mathrm{wt} . \%$ acetone alloy system with physical properties summarized in Table 1 is used. The frozen-temperature approximation with gradient $G$ and pulling velocity $V$ is imposed in the $x$-direction defined in Fig. 1. The initial temperature profile is so that the temperature at a distance equal to the solid length, i.e., the location of a planar front, is equal to the liquidus temperature $T_{L}$ at the nominal concentration. Growth competition behavior has shown to be very different in cellular, dendritic, and degenerate regimes. Here, we focus on the influence of the temperature gradient $G$ on columnar grain growth competition in a growth regime where the primary dendrite spacing $O\left(10^{2}-10^{3}\right) \mu \mathrm{m}$ is much larger than the solutal diffusion length $l_{D}=$ $D / V=14.8 \mu \mathrm{m}$. This growth regime favors the formation of highly branched hierarchical dendritic microstructures prevalent in castings. We fix the pulling velocity to $V=86 \mu \mathrm{m} \mathrm{s}^{-1}$, which is the same as the experimental value in [16]. In this growth regime, the dendrite growth orientation almost exactly coincide with the $\langle 10\rangle$ crystal axes ( $<100\rangle$ in 3D), unlike the regime studied in the previous work $[29,31]$.

The initial state consists of two grains of equal solid length in the direction of the temperature gradient positioned in the left part of the domain. The solid length is selected to be at least twice larger than the depth $\delta_{t r}$ at which tertiary branches start to grow in the diverging GB region. At smaller $G$, tertiary branching starts deeper within the GB region, and hence a larger solid length in the $x$ direction is needed. This length for various temperature gradients is characterized in section 3.1.3. As illustrated in Fig. 1, periodic boundary conditions in the $y$ direction impose a diverging GB and a converging GB in each simulation. Due to increased dendrite arm spacing, a larger domain size in the $y$ direction is needed for smaller G. Equally spaced arrays of columnar dendrites with small prescribed arm spacing are initially simulated and used as input so that stable spacing selection occurs through cell/dendrite elimination. 
No-flux boundary conditions for solute mass conservation and phase-field in the pulling direction are applied. We model the moving frame so that the solid length, i.e., the length of the mushy zone, remains constant. There exists an initial transient acceleration of the front advancement and adjustment of the grain profile to the imposed bi-crystalline orientation $\left(\alpha_{1}, \alpha_{2}\right)$. Step by step, a difference in grains front position $\delta$ develops. The steady state condition corresponds to the situation when the distance between the most advanced fronts (tips) of the two grains is almost stable, i.e., $\delta=\delta_{s t}$. The distance between the front of the two grains $\delta$ may remain almost stable or it may again go through another transient state associated with the elimination of one grain during growth competition. In all simulations, it is ensured that the growth competition reaches steady state condition. The duration that steady state condition lasts is grain/simulationsize dependent; the smaller simulation size, we observe a shorter steady state duration. Simulations in which the steady state criterion is not met, i.e., early elimination occurs, are discarded.

A comparison of $\delta_{S t}$ obtained from PF simulations and predicted by Ivantsov-Solvability solution [32] is presented in Fig. 3. The solvability condition for an isothermal parabolic tip with growth velocity of $V / \cos \alpha$ (which applies to the case of misoriented grains described in Fig. 1), gives $\rho^{2} V=D d_{0} \cos \alpha / \sigma$. We account for the temperature dependence of the capillary length [33] as $d_{0}(\Delta)=\frac{d_{0}}{1-(1-k)(1-\Delta)}$, with $\Delta=\frac{T-T_{L}}{T_{S}-T_{L}}$ being the dimensionless undercooling and $T_{L}$ (resp. $T_{S}$ ) being the liquidus (resp. solidus) equilibrium temperature at a nominal composition $c_{0}$. Assuming a locally isothermal tip, the undercooling at a fixed misorientation angle $\Delta_{\alpha}$ can be written as

$$
\Delta_{\alpha}=\frac{k}{(1-k)}\left(\frac{d_{0} V}{4 k D P^{2} \sigma \cos \alpha}-1\right)
$$

where Peclet, $\mathrm{P}$, is associated with the tip radius $\rho$ as $P=\frac{\rho V}{2 D \cos \alpha}$. $\sigma$ is the tip selection parameter and $\sigma=0.057$ is obtained from PF simulations corresponding to the anisotropy of the interface 
free energy, $\varepsilon=0.014$. The undercooling $\Delta_{\alpha}$ is obtained by solving equation 2 coupled with Ivantsov's solution for a parabolic dendrite tip operating state in 2D [32]. As a result, for a bicrystal $\left(\alpha_{1}, \alpha_{2}\right)$, the distance between the tip of the two grains can be calculated through $\delta_{S t}=$ $l_{T}\left|\Delta_{\alpha_{1}}-\Delta_{\alpha_{2}}\right|$, with $l_{T}$ being the thermal length $l_{T}=\frac{T_{L}-T_{S}}{G}$. Fig. 3 shows that PF predictions of $\delta_{S t}$ are in good agreement with the Ivantsov-Solvability solution. It also indicates that the isotropic finite-difference implementation of the PF model used in this paper can accurately resolve dendritic tips, whereas the conventional anisotropic finite-difference implementation would yield less accurate $\delta_{S t}$ values as well as dendrite growth directions artificially deviating from the crystal axes that can significantly influence GB orientation selection.

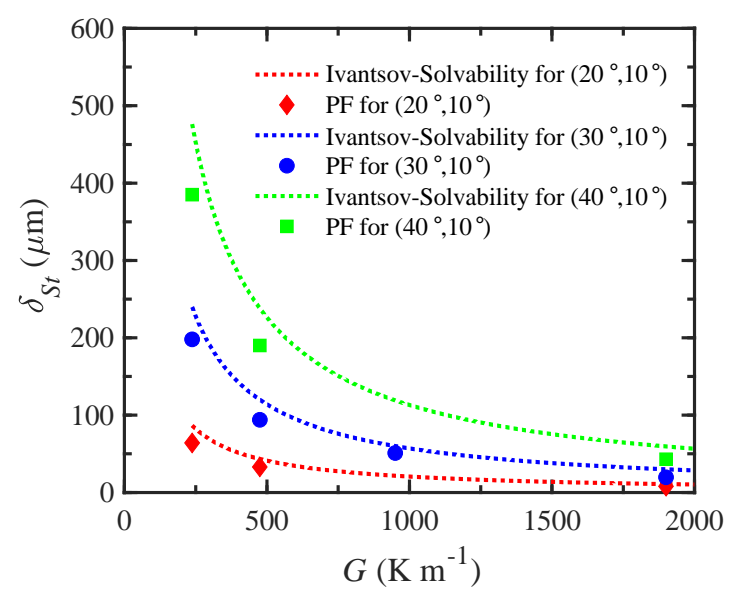

Fig. 3. (2D) Comparison of predictions of sharp-interface theory (Ivantsov solution and microscopic solvability condition) and PF simulations for the stationary distance $\delta_{S t}$ (Fig. 1) between the tip positions of the two grains for three bi-crystallographies $\left(\alpha_{1}, \alpha_{2}\right)$ corresponding to $\left(20^{\circ}, 10^{\circ}\right),\left(30^{\circ}, 10^{\circ}\right)$, and $\left(40^{\circ}, 10^{\circ}\right)$. The selection parameter $\sigma=0.057$ calculated based on PF simulations for an anisotropy $\varepsilon=0.014$ was used in the sharp-interface theory [32]. 
Large scale simulations with long steady state duration for a few selected GB bicrystallography are performed (in section 3.1.1) in order to study the GB orientation transition from FOG to GL dominance as a function of $G$. These simulations are further analyzed (in section 3.1.3) in order to characterize the GB microstructural length scales and the grain envelope. For these purposes, only steady state regions of growth competition are considered. The unsteady regions, both at the initial acceleration stage and final grain elimination stage are disregarded in the measurement of the GB angle, microstructural length scales, and the grain envelope. These simulations required a GPU memory of $\sim 33$ to 66 gigabyte and were performed on Nvidia Tesla V100 GPUs. At $G=1900 \mathrm{~K} \mathrm{~m}^{-1}$, a $\sim 3 \times 1.5 \mathrm{~mm}$ domain moving in the $x$ direction at a velocity $V=86 \mu \mathrm{m} \mathrm{s}^{-1}$ over $120 \mathrm{~s}$ is simulated. This yields a solidification length of roughly $\sim V \times t=10$ $\mathrm{mm}$ in the $x$ direction and the computing time was $\sim 7$ days. Similarly, at $G=950 \mathrm{~K} \mathrm{~m}^{-1}$, we simulate a domain of $\sim 3 \times 1.75 \mathrm{~mm}$ over $120 \mathrm{~s}$ with a computing time $\sim 8$ days. At $G=475 \mathrm{~K} \mathrm{~m}^{-1}$, simulations are conducted for a domain of $\sim[3-4] \times 2 \mathrm{~mm}$ over [120-200] s with a computing time of $\sim$ [9-23] days. For $G=237.5 \mathrm{~K} \mathrm{~m}^{-1}$ a domain of $\sim 5 \times 2.5 \mathrm{~mm}$ over $\sim[200-240] \mathrm{s}$ is simulated and the computing time was $\sim 35-40$ days. For each case, 3 to 4 simulations were performed with a variable random number seed to account for the stochasticity inherent in the incorporation of noise, and slightly different domain sizes in the aforementioned ranges were used; the average values of the GB angles are measured from these simulations.

Smaller scale simulations are performed (in section 3.1.2) to map the GB angle for the entire $\left[-45^{\circ},+45^{\circ}\right]$ orientation range of both grains $\left(\alpha_{1}, \alpha_{2}\right)$ at the two temperature gradients of $G=1900$ and $475 \mathrm{~K} \mathrm{~m}^{-1}$. Exploiting symmetries and using a uniform mapping, the whole domain can be mapped with $5^{\circ}$ steps using a total of 190 configurations, 19 of which correspond to the single crystal configurations at $\alpha_{1}=\alpha_{2}$, and 18 others, excluding $\left(0^{\circ}, 0^{\circ}\right)$, correspond to the 
symmetry line $\alpha_{1}=-\alpha_{2}$ with average GB angle of zero. Symmetries impose that the GB angle satisfies the condition $\theta\left(\alpha_{1}, \alpha_{2}\right)=-\theta\left(-\alpha_{2},-\alpha_{1}\right)$. At $G=1900 \mathrm{~K} \mathrm{~m}^{-1}$, a domain of $\sim 1500 \times$ $1615 \mu \mathrm{m}$, and at $G=475 \mathrm{~K} \mathrm{~m}^{-1}$ a domain of $\sim 2000 \times 1800 \mu \mathrm{m}$ moving in the $x$ direction at a velocity of $86 \mu \mathrm{m} \mathrm{s}^{-1}$ over, respectively, 80 and $120 \mathrm{~s}$ are simulated. Due to smaller domain sizes, steady state duration of these simulations is small. Simulations that did not reach steady state, i.e., quick elimination occurrence, were discarded. The GB angle measurements are performed on the whole GB trajectory that also observes the unsteady state variations of $\delta$. For the symmetry line $\alpha_{1}=-\alpha_{2}$, a few selected configurations were checked to ensure the divergence of the average GB angle of up to ten simulations with different microscopic fluctuations to $0^{\circ}$, and hence for saving computational cost, in the GB maps, value $\theta=0^{\circ}$ was used for all the configuration corresponding to the symmetry line. Each simulation required about [5.5-7.5] gigabyte of GPU memory and was performed on Nvidia Tesla p100 GPUs. Fixing the time step to $\Delta t=\frac{0.8 \Delta x^{2}}{4 D}$ each simulation related to the $G=475 \mathrm{~K} \mathrm{~m}^{-1} \mathrm{~GB}$ map took about $200 \mathrm{hrs}$, and each simulation at $G=$ $1900 \mathrm{~K} \mathrm{~m}^{-1}$ took about $120 \mathrm{hrs}$; hence, a total computing time of greater than $64,000 \mathrm{hrs}$ for more than 400 smaller-scale simulations was incurred.

Table 1. Materials, process, and simulation parameters used in PF simulations

\begin{tabular}{lll}
\hline Variable & Name [unit] & Value \\
\hline Nominal composition in acetone & $c_{0}[\mathrm{wt} \%$ Ace. $]$ & 1.3 \\
Diffusion coefficient & $D\left[\mu \mathrm{m}^{2} \mathrm{~s}^{-1}\right]$ & $1270[34]$ \\
Partition coefficient & $k$ & $0.1[34]$ \\
Liquidus slope & $m\left[\mathrm{~K} \mathrm{wt.} \%^{-1}\right]$ & $-3.02[35]$ \\
Melting temperature of pure $\mathrm{SCN}$ & $T_{M}[\mathrm{~K}]$ & $331.24[35]$ \\
Liquidus temperature at $c_{0}$ & $T_{L}[\mathrm{~K}]$ & 327.314 \\
Gibbs Thomson coefficient & $\Gamma[\mathrm{K} \mathrm{m}]$ & $6.4 \times 10^{-8}[16]$ \\
Anisotropy coefficient & $\varepsilon$ & 0.014 \\
Selection parameter & $\sigma$ & 0.057
\end{tabular}


Temperature gradient

Isotherm velocity

Interface width

Capillarity length

Mesh size

$\begin{array}{ll}G\left[\mathrm{~K} \mathrm{~m}^{-1}\right] & 237.5,475,950, \\ V\left[\mu \mathrm{m} \mathrm{s}^{-1}\right] & 86 \\ W\left[\mathrm{~d}_{0}\right] & 110 \\ d_{0}[\mathrm{~nm}] & 1.81 \\ \Delta x[\mathrm{~W}] & 1.2(\text { or } 0.24 \mu \mathrm{m})\end{array}$

\subsection{Cellular automaton simulations}

The cellular automaton approach and its applications to material forming processes have been largely detailed in the literature. The modelling of grain growth evolution during solidification processes based on CA resolution was initially proposed by Gandin and Rappaz $[23,36]$. This approach relates to the use of a regular grid of small square cells, which is superimposed to the domain of interest. Grain growth is then modeled at cell scale using a polygonal (resp. polyhedral) shape in 2D with directional axes corresponding to crystallographic $\langle 10\rangle$ directions assumed as the growth direction of dendrite tips. This shape aims to simulate the development of dendrite arms inside each cell during cooling for given growth velocities, $v^{\langle 10\rangle}$, imposed at the four $\langle 10\rangle$ tips. Growth velocity can be computed using the Ivantsov relation and a stability criterion with a chosen value of the selection parameter $\sigma$ as proposed by Kurz et al. [37]. However, the solution of these equations is usually correlated as a simple power-law relation as proposed in the present application. This growing stage needs to be based on the knowledge of the temperature field evolution and its value at dendrite tips. The computation of the temperature evolution may be developed separately at a larger scale by solving the energy conservation equation using a finite elements method. However, this temperature evolution could also be imposed using fixed values of the temperature gradient, $G$, and the pulling velocity, $V$ as done in the present PF simulations. This approach was similarly applied by Pineau et al. [8] for comparing CA with PF simulation results. 
An application of the CA method is proposed thereafter in order to model grain growth for the same alloy investigated by PF simulations previously detailed in Table 1 and using identical cooling conditions. In the present 2D CA, a computational grid composed of square cells of identical size, $l_{C A}$, is used. Solidification is developed onto a large rectangular CA computational domain schematized in Fig. 4. A constant temperature gradient $G$ is imposed on the whole domain. At the initial time, the left-hand side (i.e., $x=0 \mathrm{~m}$ ) is at the liquidus temperature, $T_{L}$. A homogeneous cooling rate, $\dot{T}=-G \cdot V$, is then applied on the whole domain leading to isotherms moving with constant horizontal velocity, $V$. The solidification starts on the left-hand side at $t=$ $0 \mathrm{~s}$ by assuming a negligible nucleation undercooling for the first row of cells (black contour) associated with grain 1 (blue) and 2 (red) sharing the height of the left boundary. A GB groove also forms as a result of the competition between the development of the two grains in the $x$ direction. Far from the GB groove, grain 1 (resp. 2) of angle $\alpha_{1}$ (resp. $\alpha_{2}$ ) develops with growth velocity $v_{\alpha_{1}}$ (resp. $v_{\alpha_{2}}$ ) of horizontal component $v_{x_{1}}\left(\right.$ resp. $v_{x_{2}}$ ). The solidification front position $x_{1}$ (resp. $x_{2}$ ) of temperature $T_{1}\left(\right.$ resp. $T_{2}$ ) is associated with the [10] direction of grain 1 (resp. grain 2) and corresponds to a growth undercooling $\Delta T_{1}$ (resp. $\Delta T_{2}$ ). The solidification fronts associated to grains 1 and 2 reach a steady state after a sufficient cooling duration when both velocities $v_{\alpha_{1}}$ and $v_{\alpha_{2}}$, have a stabilized $x$-component value equal to the isotherm velocity, $v_{x_{1}}=$ $v_{x_{2}}=V$. Within the groove, cells at the boundary with the liquid of grain 1 (resp. grain 2) have undercooling higher than $\Delta T_{1}$ (resp. $\Delta T_{2}$ ) and hence higher velocity than $v_{\alpha_{1}}$ (resp. $v_{\alpha_{2}}$ ) for all $\langle 10\rangle$ directions.

The domain is thus dimensioned with sufficiently large sizes in $(x, y)$ directions in order to reach steady state development of the GB before full achievement of solidification. In steady condition, 
the difference between solidification front positions, $\delta$, is stable and equal to the previously defined $\delta_{S t}$ value. The time evolution of $\delta$ is detailed in the Appendix in order to provide an estimation of the time required to achieve steady state regime in CA simulation considering $\delta_{S t}$ dependence on $\left(\alpha_{1}, \alpha_{2}\right)$ angles. In the present application, a correlation of the dendrite tip growth kinetics [31] was done with material properties associated to succinonitrile - $1.3 \mathrm{wt} \%$ acetone (Table 1). The set of $(\Delta T, v)$ solutions in the interval $[0,2]^{\circ} \mathrm{C}$ was well correlated with the following power law relation:

$$
\begin{aligned}
& v=A \Delta T^{n} \\
& \text { with: } \quad A=9.688 \mu \mathrm{m} \mathrm{s}^{-1} \mathrm{~K}^{-\mathrm{n}} \\
& n=3.88
\end{aligned}
$$

Equation 3 leads to $v_{\alpha 1}=A \Delta T_{1}^{n}$ (resp. $v_{\alpha 2}=A \Delta T_{2}^{n}$ ) for the growth velocity at the dendrite tips of the stable fronts associated with the [10] direction of grain 1 (resp. grain 2). This growth kinetics is nothing but the 2D Ivantsov steady solute diffusion regime in the liquid ahead of a parabola together with the solvability criterion with a constant value of the tip selection parameter $\sigma$ and a constant far field nominal composition $c_{0}$. In fact, one perfectly retrieves the Ivantsov-Solvability curves reported in Fig. 3 when using the present correlation. In present CA simulations, composition variations are not present, which is justified for the well-established dendritic regime under study (primary dendrite spacing is much larger than the solutal diffusion length) and the absence of macrosegregation induced by long range transport (e.g., due to thermosolutal convection of the liquid phase).

The CA growth algorithm consists of a time integration of the growth kinetics in all $\langle 10\rangle$ crystal directions, i.e., along the main growth directions of the dendrite arms, for each CA cell located at the growth front. The evolution of the CA grain envelopes at the GB locations, in the 
vicinity of the groove, can mimic the dynamics of the secondary and tertiary branching as the local undercooling and hence the local velocity along the secondary and tertiary branches are higher than the corresponding steady values. The grove is the result of competition between the $[0 \overline{1}]$ secondary branches of the top grain and the [01] secondary branches of the bottom grain, but also the [10] tertiary branches of both grains. The generation of these branches is proportional to the cell size and the same growth kinetics law (equation 3) is used for all branches (primary, secondary and tertiary). Hence, velocity is solely function of the temperature, assuming Ivantsov solution holds with constant far field composition given by the alloy composition. In other words, the effect of the composition variations at the GB that play a role at the scale of the secondary arm spacing is not considered.

In practice, the rectangular computational domain is composed of 1200 and 3600 cells in the $x$ and $y$ directions, respectively. Consequently, the domain dimensions depend on the cell size. It is worth noting that no periodic boundary conditions are applied to the top and bottom boundaries of the domain, so only one converging or one diverging bi-crystalline configuration is studied for each simulation. For each simulation, the typical CPU time using a single node on a desktop computer (no parallel computation) is only in minutes. The dimensions of the domains are adjusted to reach $\delta_{S t}$ and to have a sufficiently long GB of constant angle $\theta$ to be extracted from the simulations. The width of the domain is selected so that the bi-crystal survives at the end of the simulation domain, and there is no need to discard any result that would end with a single crystal. 


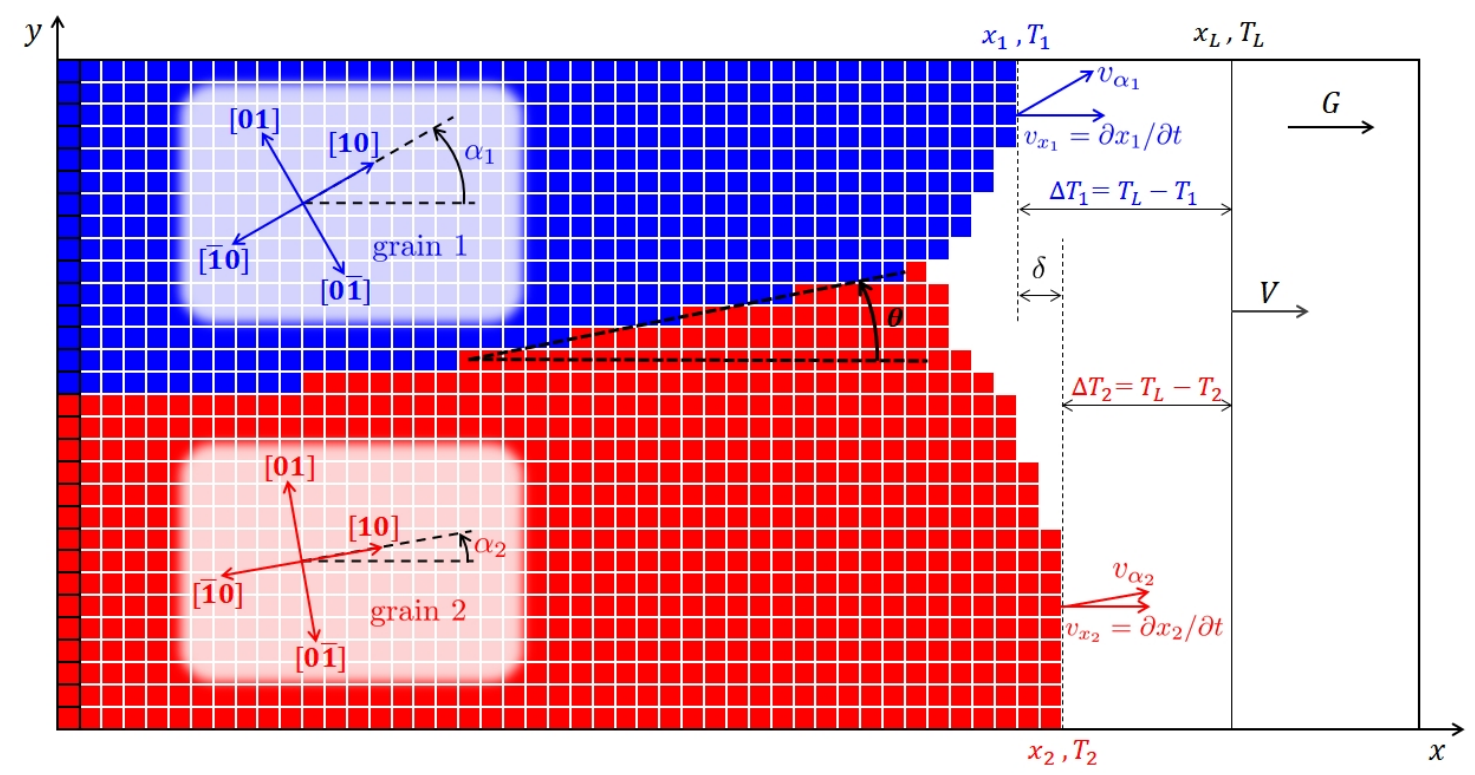

Fig. 4. CA growth bi-crystal scheme with constant temperature gradient, $G$, isotherm velocity, $V$, liquidus temperature, $T_{L}$, grain front position $x_{1 / 2}$, with temperature $T_{1 / 2}$, undercooling $\Delta T_{1 / 2}$, and velocity $v_{\alpha_{1 / 2}}$ for grains 1 and 2 of corresponding orientation $\alpha_{1 / 2}$, as well as the difference between solidification front positions, $\delta=\left|x_{2}-x_{1}\right|$. After a sufficiently long solidification time, a steady state regime is achieved leading to a stable value for $\delta=\delta_{S t}$ and a steady GB with orientation angle $\theta$. The first row of cells highlighted with black contours identifies the initial distribution of the grains in the domain.

\section{Results}

\subsection{PF simulations}

\subsubsection{GB orientation transition from FOG to GL dominance}

Fig. 5 shows the map of the microstructure and orientation selection as a function of $G$ for three different bi-crystallography $\left(20^{\circ}, 10^{\circ}\right),\left(30^{\circ}, 10^{\circ}\right)$, and $\left(40^{\circ}, 10^{\circ}\right)$. The microstructure represents the history of the computed growth. The orange dashed lines in Fig. 5 follow the direction of the diverging GBs. From the microstructure map, it is evident that the evolution of the diverging GB is determined by competition between secondary and tertiary branches to invade the GB region. At higher temperature gradient $G=1900 \mathrm{~K} \mathrm{~m}^{-1}$, the GB region is primarily occupied 
by branches of the most misoriented grain, which is grain 1 for the diverging GBs considered here, hence, corresponding to FOG. As shown in Fig. 5, secondary branches of the unfavorably oriented grain grow long, and at regular instances give rise to stable tertiary branches. The secondary branches of the most favorably oriented grains (here grain 2) grow minimally and are regularly blocked from growing a stable tertiary branch. As a result, the diverging GB angle follows the direction of the crystal angle of the most favorably oriented grain.

At lower temperature gradients $G=475$ and $237.5 \mathrm{~K} \mathrm{~m}^{-1}$, the diverging GB region of $\left(30^{\circ}, 10^{\circ}\right)$ and $\left(20^{\circ}, 10^{\circ}\right)$ bi-crystallography is occupied by secondary branches from both grains and they both give rise to stable tertiary branches. In fact, development of the secondary arms into the GB region alters the GB angle and the formation of stable tertiary branches maintains and feeds that adjustment. For the smaller $G$, the secondary branches of the most favorably oriented grain develop more into the GB region and tend to give rise to more instances of stable tertiary branches, thereby causing a departure of the GB angle from the FOG prediction. For the $\left(30^{\circ}, 10^{\circ}\right)$ and $\left(20^{\circ}\right.$, $10^{\circ}$ ) configurations that form well-developed dendritic structures with distinct primary and secondary branches, we find, by measuring the GB orientations, that with decreasing $G$ at fixed bi-crystallography, a transition from FOG dominance $\left(10^{\circ}\right)$ to GL dominance (bisector of the bicrystallography) occurs, as shown in Fig. 6 a where the GL limits are also plotted $\left(15^{\circ}\right.$ at $\left(20^{\circ}, 10^{\circ}\right)$, $20^{\circ}$ at $\left(30^{\circ}, 10^{\circ}\right)$ and $25^{\circ}$ at $\left(40^{\circ}, 10^{\circ}\right)$ configurations). For the $\left(20^{\circ}, 10^{\circ}\right)$ configuration, by decreasing $G 8$ fold from 1900 to $237.5 \mathrm{~K} \mathrm{~m}^{-1}$, the average diverging GB angle, $\overline{\theta_{D}}$, varied from $10^{\circ}$ to $14^{\circ}$. Similarly, for the $\left(30^{\circ}, 10^{\circ}\right)$ configuration, $\overline{\theta_{D}}$ varied from $10^{\circ}$ to $16^{\circ}$ as $G$ decreases from 1900 to $237.5 \mathrm{~K} \mathrm{~m}^{-1}$. The full GL limit is expected to be observed for even lower temperature gradients that were not investigated because of the high computational cost. 
The second observation is that, at lower temperature gradients $G=475$ and $237.5 \mathrm{~K} \mathrm{~m}^{-1}$, at a constant $G, \theta_{D}$ is a non-monotonic function of the difference in dimensionless undercooling $\left|\Delta_{\alpha_{1}}-\Delta_{\alpha_{2}}\right|$, and hence distance $\delta_{S t}$ between the dendrite tips of the two grains imposed by the bicrystallography $\alpha$. Because for all configurations in Fig. 5, the converging GB gives constant $\theta_{C} \approx$ $10^{\circ}$, the rate of elimination of the most misoriented grain defined by $\theta_{D}-\theta_{C}$ follows a similar trend as $\theta_{D}$ versus undercooling $\left|\Delta_{\alpha_{1}}-\Delta_{\alpha_{2}}\right|$. This observation is plotted in Fig. 6b. A similar observation was made in Ref. [29] for $0.4 \mathrm{wt} \%$ composition, $V=25 \mu \mathrm{m} \mathrm{s}^{-1}$ and $G=30000-$ $90000 \mathrm{~K} \mathrm{~m}^{-1}$ showing the failure of the assumption that the elimination rate of the misoriented grain always increases with this undercooling difference [29]. For the $\left(40^{\circ}, 10^{\circ}\right)$ bi-crystallography and smaller $G=237.5-475 \mathrm{~K} \mathrm{~m}^{-1}$, the steady state region was shorter due to limitations imposed by simulation size. Therefore, for consistency of the map, only a portion of the solidification history is shown in Fig. 5 for all G. Unlike for $\left(20^{\circ}, 10^{\circ}\right)$ and $\left(30^{\circ}, 10^{\circ}\right)$, where the secondary and primary arms (i.e., tertiary arms at the GB region) have clearly distinct features, for the $\left(40^{\circ}, 10^{\circ}\right)$ configuration, the secondary and primary arms of the most misoriented grain $40^{\circ}$ are almost indistinguishable and exhibit similar features e.g., arm spacing, and tip curvature (and undercooling). This is referred to as a degenerate microstructure.

Hence, for the $\left(40^{\circ}, 10^{\circ}\right)$ degenerate configuration, the competition is in fact between sturdy primary arms of the unfavorably oriented grain and weak secondary arms of the most favorably oriented grain, making the most misoriented grain the winner in growth competition in steady state. This can be explained by considering the limiting case of the $\left(45^{\circ}, 10^{\circ}\right)$ bi-crystallography. In this configuration, symmetry is imposed by the $45^{\circ}$ grain so that its primary and secondary arms are the same and the grain grows while maintaining a flat front with all the dendrite tips 
approximately sharing the same undercooling. Due to this flat profile, there is little to no open GB region between the two grains and hence competition is limited. Therefore, in steady state, grain 1 and 2 both co-exist while forming two GB angles with $\theta=10^{\circ}$. This explains the nonmonotonicity of the $\theta$ versus $\left|\Delta_{\alpha_{1}}-\Delta_{\alpha_{2}}\right|$ curve in Fig. $6 \mathrm{~b}$. For the $\left(45^{\circ}, 10^{\circ}\right)$ bi-crystallography, elimination of the most misoriented grain can occur only when $\delta_{S t}$ is larger than the depth $\delta_{t r}$ at which a stable tertiary branch can start on the secondary arms of the most favorably oriented grain. If this condition holds, as soon as growth reaches a steady state, the most favorably oriented grain gives rise to a stable tertiary branch at a location ahead of the most misoriented grain front, thereby dictating the GB orientation. 


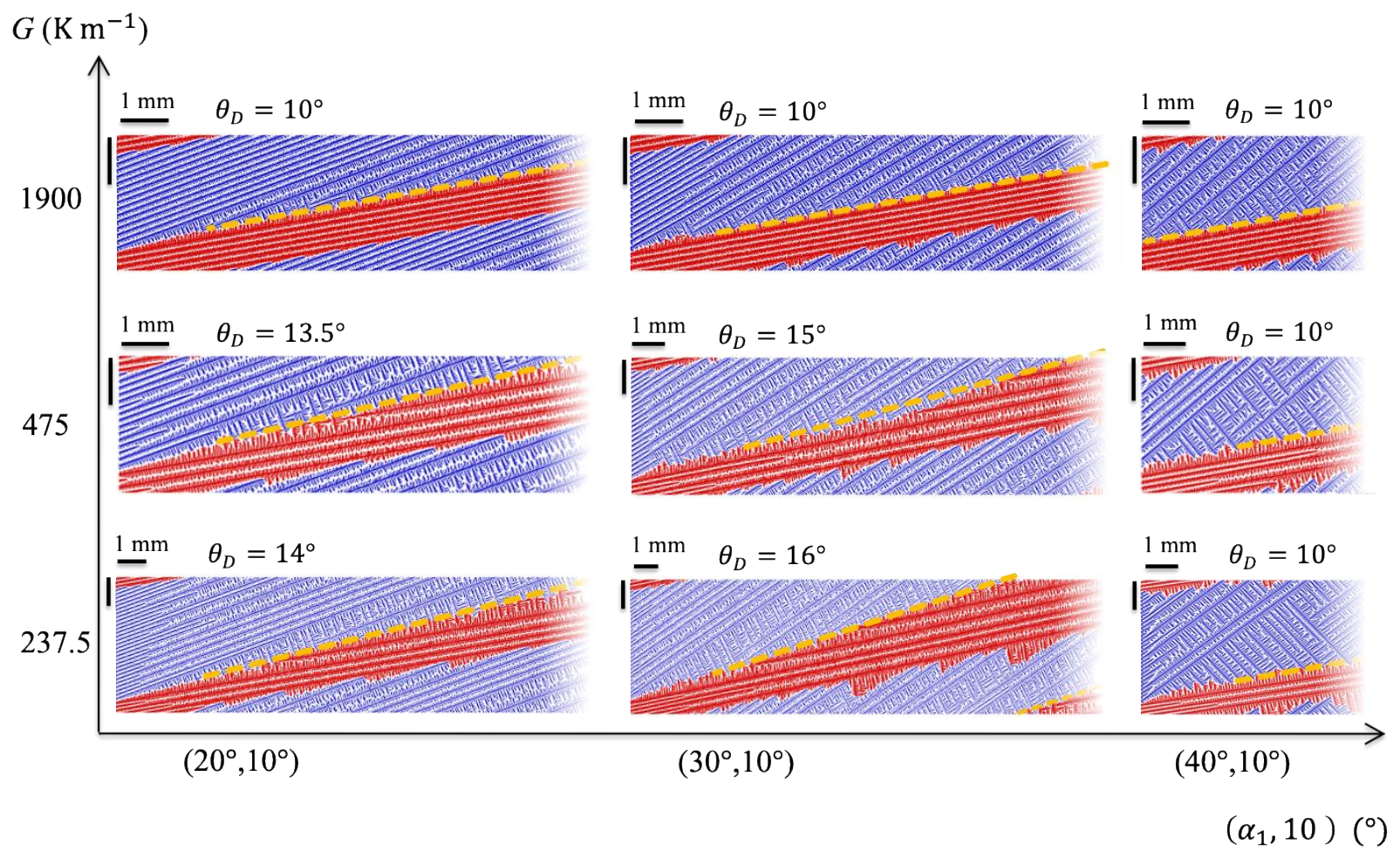

Fig. 5. Phase-field simulation results showing dendritic microstructure evolution and GB orientation selection for a SCN-1.3 wt.\% Ace alloy directionally solidified for a pulling velocity $V=86 \mu \mathrm{m} \mathrm{s}^{-1}$ and different $G$ and bi-crystallographies $\left(\alpha_{1}, \alpha_{2}\right)$ with fixed $\alpha_{2}=10^{\circ}$. The orange dashed lines follow the average direction of the diverging GBs, $\theta_{D}$, and, for all bicrystallographies, the converging GB has approximately the same orientation $\theta_{C} \approx 10^{\circ}$. Note that the $1 \mathrm{~mm}$ scale bar is shorter in (c) than in (a) and (b). 
(a)

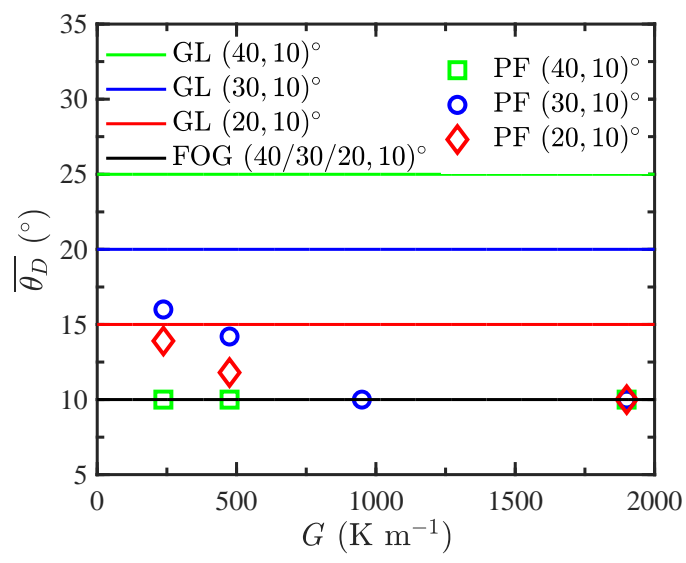

(b)

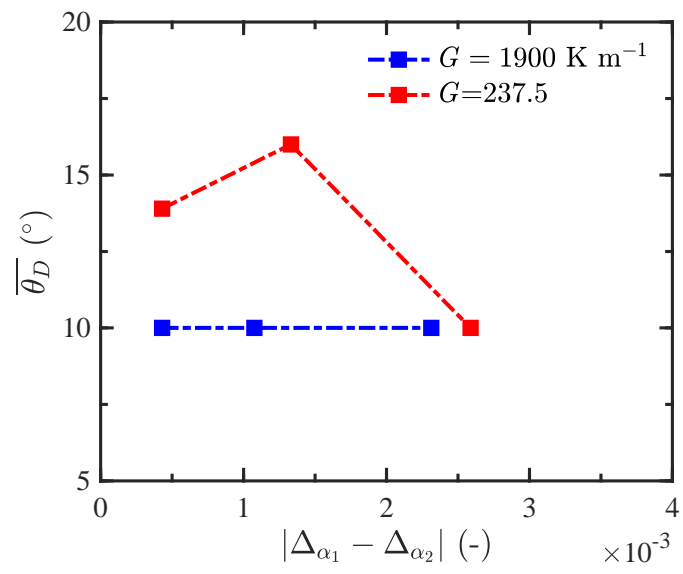

Fig. 6. Phase-field simulation results showing a transition from FOG to GL with decreasing temperature gradient. (a) Average diverging GB orientation angle, $\overline{\theta_{D}}$, vs $G$ for different bicrystallographies $\left(20^{\circ}, 10^{\circ}\right),\left(30^{\circ}, 10^{\circ}\right)$ and $\left(40^{\circ}, 10^{\circ}\right)$, and $(\mathrm{b}) \overline{\theta_{D}}$, vs steady-state undercooling difference, $\left|\Delta_{\alpha_{1}}-\Delta_{\alpha_{2}}\right|$, in the FOG high-gradient $\left(G=1900 \mathrm{~K} \mathrm{~m}^{-1}\right)$ and GL low-gradient ( $\left.G=237.5 \mathrm{~K} \mathrm{~m}^{-1}\right)$ dominant regimes. Each point from left to right along the $\left|\Delta_{\alpha_{1}}-\Delta_{\alpha_{2}}\right|$ axis corresponds to $\left(20^{\circ}, 10^{\circ}\right),\left(30^{\circ}, 10^{\circ}\right)$, and $\left(40^{\circ}, 10^{\circ}\right)$, respectively.

\subsubsection{GB orientation selection maps}

Fig. 7 shows the GB orientation selection map for the two temperature gradients $G=$ $1900 \mathrm{~K} \mathrm{~m}^{-1}$ and $G=475 \mathrm{~K} \mathrm{~m}^{-1}$ in $\left(\alpha_{1}, \alpha_{2}\right)$ plane. While the small sample size limits somewhat the resolution of the map, the transition from FOG to GL dominance with decreasing $G$ is clearly seen. By comparing Fig. 7 to Fig. 2, it is evident that the PF map for $G=1900 \mathrm{~K} \mathrm{~m}^{-1}$ looks similar to the FOG map while the PF map for $G=475 \mathrm{~K} \mathrm{~m}^{-1}$ looks similar to the GL map. This behavior confirms the previously reported finding using CA simulations [8]. Further comparison between PF-FOG and PF-GL is performed by computing the difference between $\theta$-values for a 
given couple of $\left(\alpha_{1}, \alpha_{2}\right), \Delta \theta$, e.g. $\left|\theta_{P F}\left(\alpha_{1}, \alpha_{2}\right)-\theta_{G L}\left(\alpha_{1}, \alpha_{2}\right)\right|$, for the whole map as well as the first quarter of the map, i.e., region with $\alpha_{1}$ and $\alpha_{2} \geq 0$. The first quarter of the map corresponds to the bi-crystallography of particular interest, which contain one diverging and one converging GB. The arithmetic mean difference $m$, and standard deviation $s$ are calculated based on the number of $\Delta \theta$ values. For better analysis, the median $M$, first quartile $Q_{1}$ and third quartile $Q_{3}$ were also extracted in order to avoid large influence of extreme values (as it is present in the arithmetic mean). These quantities are listed in Table 2 for $G=1900$ and $475 \mathrm{~K} \mathrm{~m}^{-1}$. Table 2 shows at $G=$ $1900 \mathrm{~K} \mathrm{~m}^{-1}$, PF results are remarkably closer to the FOG criterion rather than GL criterion. At $G=475 \mathrm{~K} \mathrm{~m}^{-1} \mathrm{PF}$ results are closer to the GL considering both the whole map and the first quarter of the map. As it was shown in section 3.1.1, by further decreasing the temperature gradient to $G=237.5 \mathrm{~K} \mathrm{~m}^{-1}$, the GB orientation further converges towards the GL limit. However, the computational cost at lower $G$ is higher. Hence, the map was only created for $G=475 \mathrm{~K} \mathrm{~m}^{-1}$ that already reveals the tendency towards GL.

For the single configuration $\left(30^{\circ}, 10^{\circ}\right)$, statistical analysis is performed to ensure that FOG dominance at $G=1900 \mathrm{~K} \mathrm{~m}^{-1}$ and GL dominance at $G=475 \mathrm{~K} \mathrm{~m}^{-1}$ is not affected by the inherent stochasticity of microscopic fluctuations. Fig. 8 shows the grain boundary trajectory for 20 simulations with different random number seeds. For $G=1900 \mathrm{~K} \mathrm{~m}^{-1}$ both converging and diverging GB followed an angle of $10^{\circ}$ as predicted by the FOG criterion, $\theta_{D / C, F O G} \approx 10^{\circ}$ (Fig. 8a). For $G=475 \mathrm{~K} \mathrm{~m}^{-1}$ both diverging and converging GB angles show deviation from FOG and tendency towards GL where the maximum GL dominance corresponds to $\theta_{D}=15.35^{\circ}$ and $\theta_{C}=$ $8.37^{\circ}$ and the minimum GL dominance corresponds to $\theta_{D}=11.10^{\circ}$ and $\theta_{C}=9.36^{\circ}$ (Fig. $8 \mathrm{~b}$ ). For 
this $G$, all ten simulations exhibit grain elimination even though at a slower rate than the GL limit corresponding to $\theta_{D, G L}=20^{\circ}$ and $\theta_{C, G L}=0.27^{\circ}$.

As mentioned in section 2.1, due to the limited system size, these simulations have a short steady state duration, and the GB angle measurements were performed on the whole GB trajectory that also contains a non-steady-state regime where $\delta$ is not constant. In section 3.1.1, it was shown that for $\left(40^{\circ}, 10^{\circ}\right)$ and $G=475 \mathrm{~K} \mathrm{~m}^{-1}, \theta_{D} \approx \theta_{C} \approx 10^{\circ}$ in steady state. During the non-steadystate regime, due to the large fluctuations of $\delta, \delta$ can at times become larger than the depth at which a stable tertiary branch can occur on the secondary arms of the most favorably or unfavorably oriented grain. In that case, elimination of the unfavorably oriented grain occurs outside of steady state and influences the GB angle. Hence, unlike in the steady state simulations of section 3.1.1 (with $G=475 \mathrm{~K} \mathrm{~m}^{-1}$ and degenerate configuration $|\alpha| \rightarrow 45^{\circ}$ at a diverging or converging GB), in the PF GB map of Fig. 7b presented in this section, we do not necessarily observe $\theta_{D}=\theta_{C}=\theta_{F O G}$ due to non-steady-state growth effects. 

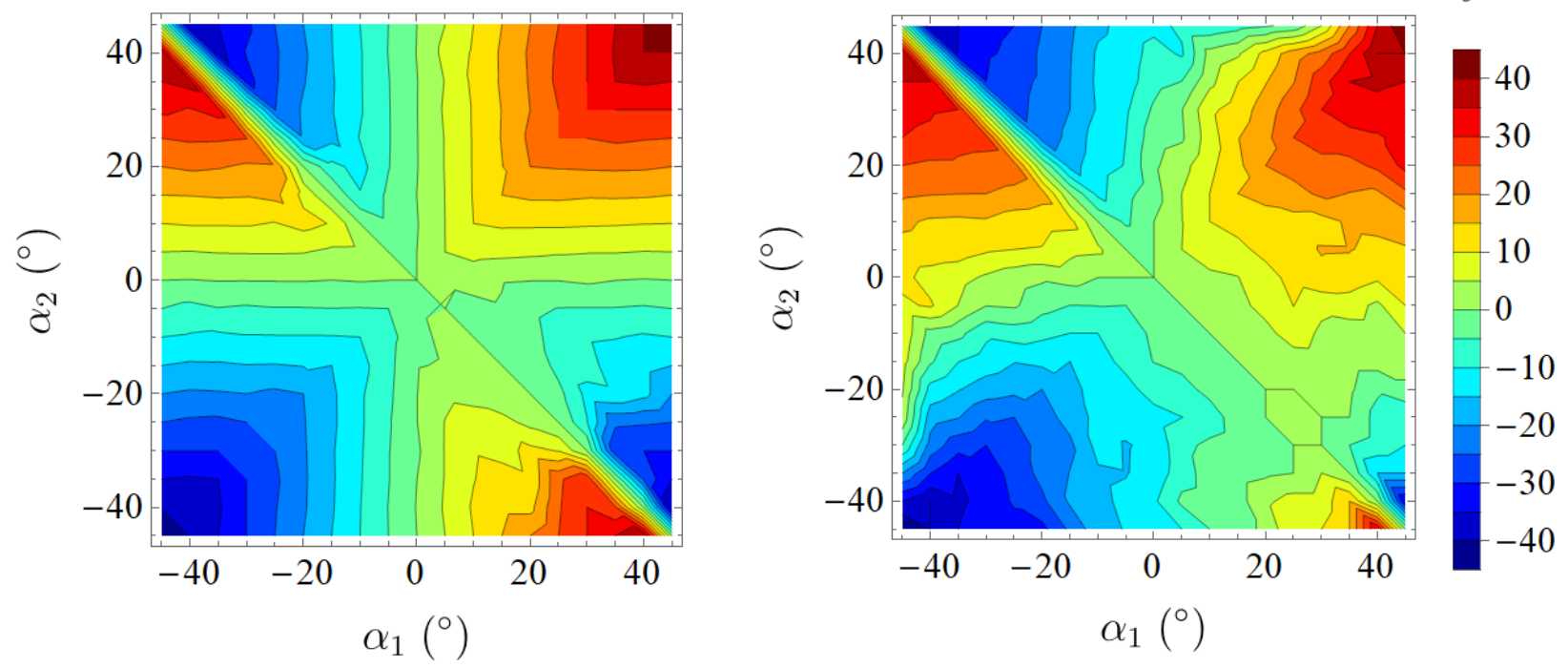

Fig. 7. GB orientation selection maps (following the same notation as Fig. 2) predicted by PF simulations for (a) $G=1900 \mathrm{~K} \mathrm{~m}^{-1}$, and (b) $G=475 \mathrm{~K} \mathrm{~m}^{-1}$.
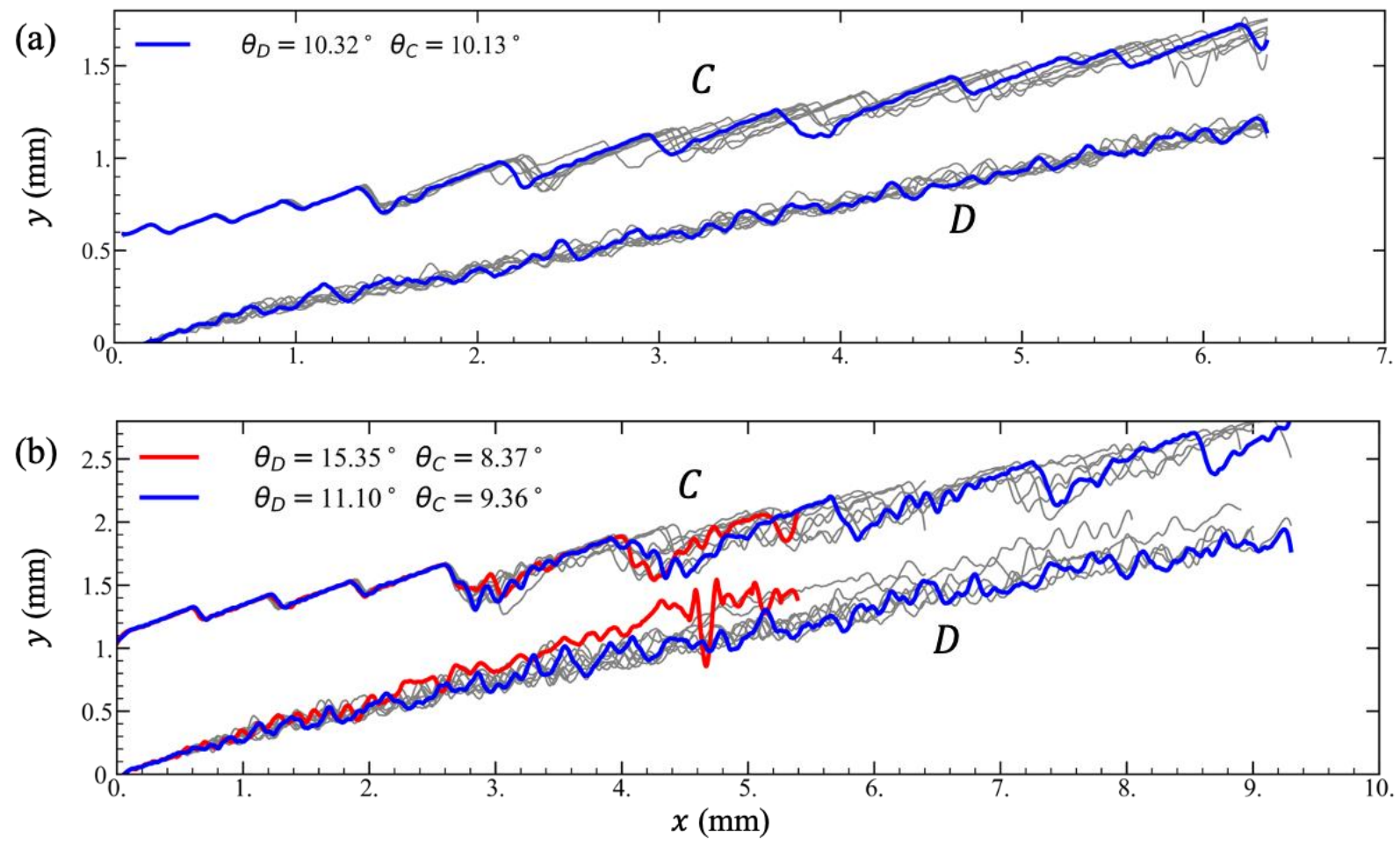
Fig. 8. GB trajectories of ten PF simulations of fixed $\left(30^{\circ}, 10^{\circ}\right)$ bi-crystallography with different initial random number seeds and hence different histories of microscopic fluctuations depicted by gray lines, the bottom and top one being respectively, the diverging $(D)$ and the converging (C) GB trajectories for (a) FOG $\left(G=1900 \mathrm{~K} \mathrm{~m}^{-1}\right.$ ) where $\theta_{D}=10.32^{\circ}$ and $\theta_{C}=10.13^{\circ}$ represent the angles of the linear fits to an arbitrary set of $D / C$ GB trajectories (blue lines), and (b) GL dominance $\left(G=475 \mathrm{~K} \mathrm{~m}^{-1}\right.$ ) where linear fits of GB trajectories yield $\theta_{D}=15.35^{\circ}$ and $\theta_{C}=8.37^{\circ}$ (red lines) and $\theta_{D}=11.10^{\circ}$ and $\theta_{C}=9.36^{\circ}$ (blue lines) corresponding to simulations with maximum and minimum GL dominance, respectively.

Table 2. Statistical study of the difference between PF-FOG, and PF-GL selection maps with mean $m$, standard deviation, $s$, first quartile, $Q_{1}$, median, $M$, and third quartile, $Q_{3}$ at $G=1900$ and $475 \mathrm{~K} \mathrm{~m}^{-1}$.

\begin{tabular}{|c|c|c|c|c|}
\hline $\begin{array}{c}G \\
\left(\mathrm{~K} \mathrm{~m}^{-1}\right)\end{array}$ & Region & $\begin{array}{c}\left(m\left[^{\circ}\right], s\left[^{\circ}\right]\right) \\
\left(Q_{1}\left[^{\circ}\right], M\left[^{\circ}\right], Q_{3}\left[^{\circ}\right]\right.\end{array}$ & FOG & GL \\
\hline 1900 & $-45^{\circ} \leq \alpha_{1} \& \alpha_{2} \leq+45^{\circ}$ & $\mathrm{PF}$ & $(1.11,2.62)$ & $(11.37,10.29)$ \\
& & & $0,0.29,0.76$ & $3.40,8.08,17.47$ \\
\hline 475 & $-45^{\circ} \leq \alpha_{1} \& \alpha_{2} \leq+45^{\circ}$ & $\mathrm{PF}$ & $(7.46,8.76)$ & $(5.37,6.44)$ \\
& & & $2.10,4.81,9.45$ & $0.78,3.01,8.05$ \\
\cline { 2 - 5 } & $\alpha_{1} \geq 0 \& \alpha_{2} \geq 0$ & $\mathrm{PF}$ & $(6.80,8.01)$ & $(4.14,4.32)$ \\
& & & $2.12,4.86,8.47$ & $0.65,2.89,6.00$ \\
\hline
\end{tabular}

3.1.3 Characterization of the microstructural length scales and grain envelope at the diverging GB

Fig. 9 shows sample retrieved instances of the start of stable tertiary branching and their corresponding growth history (as introduced in Fig. $1 \mathrm{~b}$ and c) at various $G=237.5,475$ and $1900 \mathrm{~K} \mathrm{~m}^{-1}$ and fixed non-degenerate bi-crystallography $\left(30^{\circ}, 10^{\circ}\right)$. From Fig. 9, a few observations can be made. Fig. 9 again demonstrates the transition from FOG to GL dominance by decreasing $G$; for $G=1900 \mathrm{~K} \mathrm{~m}^{-1}$ stable tertiary branches (circled by green and yellow at the origin) only form on grain 1 , while for $G=237.5$, and $475 \mathrm{~K} \mathrm{~m}^{-1}$ stable tertiary arms grow on both grain 1 and 2. It also shows that $\delta_{t r}$ increases as $G$ decrease. This behavior is further quantified in Fig. 10. Another observation is that the farther down a dendrite tip at the location $Z_{1 / 2, i}$, the longer secondary branches of length $L_{1 / 2, i}$ with wider spacing $\Lambda_{1 / 2, i \& i+1}^{(2)}$ exist. Later in Fig. 12, we quantify this behavior in detail. For smaller $G$, larger $\delta_{t r}$ corresponds to a location deeper down 
the primary tip, $Z_{1 / 2, i=2}$, and the diverging GB is constructed from the impingement of the secondary arms at that location; hence, for smaller $G$ s, GBs with larger secondary arm spacings form, which is qualitatively evident in Fig. 9.

As demonstrated in Fig. 9b, at the diverging GB, at times when the primary trunk resulting from tertiary branching on grain 1 gets hindered by a secondary arm ahead (illustrated in the yellow frame), the neighboring tertiary branch on grain 2 (circled by yellow) finds the opportunity to develop further and stabilize. The frequent blockage of tertiary arms of grain 1 by the secondary arms ahead (as visible in the growth history) leads to the wandering of the growing arm to find alternative stable spacings; this behavior further gets reflected in the variation of primary spacing selection in the GB region of the bi-crystal visible here and later quantified in Fig. 11. In addition, as seen in Fig. 9c, when younger primary trunks with weak secondary branches happen to grow in the vicinity of grain 2 (illustrated in the yellow frame), tertiary branches of grain 2 (circled by yellow) find the opportunity to grow and stabilize unhindered by the opponent (younger) branches. Formation of these young (weak) trunks that favor a GL dominated GB angle can be facilitated through the larger freedom in stable spacing selection at smaller temperature gradients as qualitatively observed here and further quantified in Fig. 10 and 11. The length scale that can best bridge $\mathrm{PF}$ and $\mathrm{CA}$ prediction of the GB angle and quantitatively reproduce transition from FOG to GL using CA simulations is later investigated in section 3.1.4. 
(a)

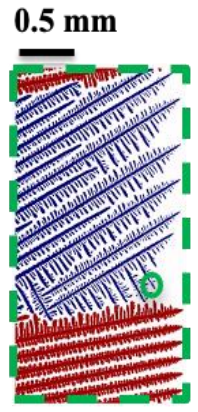

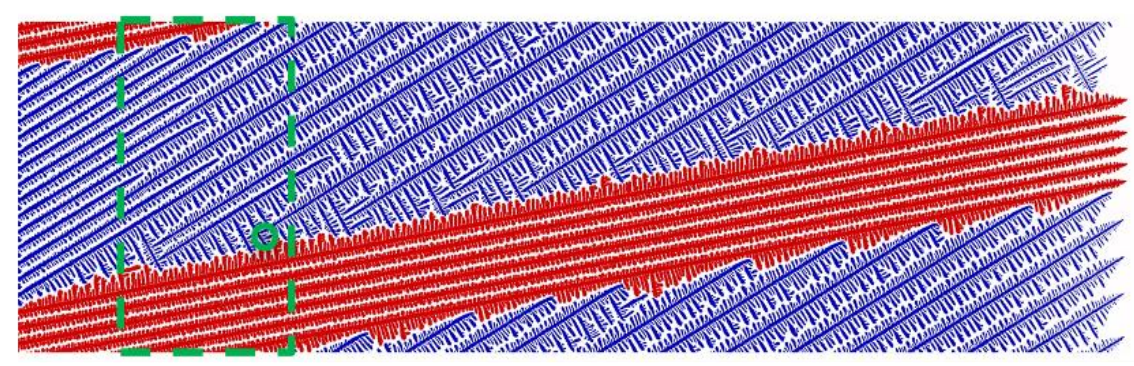

(b)
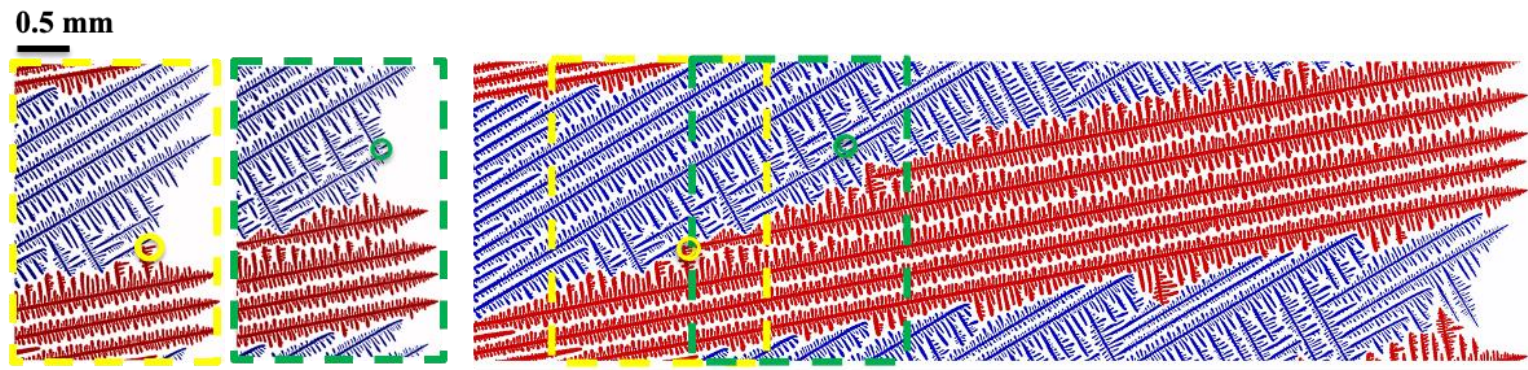

(c)

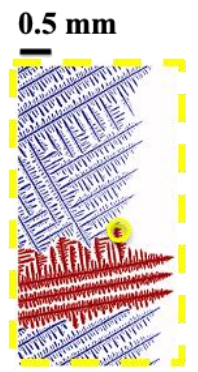

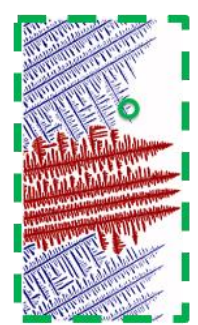

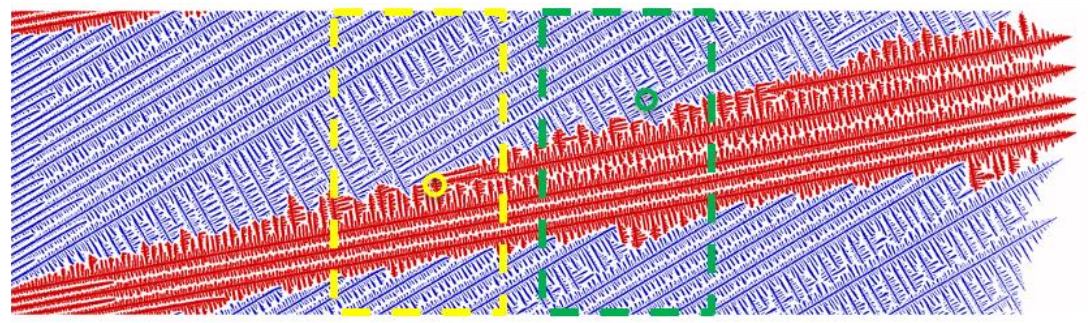

Fig. 9. Sample instances of tertiary branching events starting on grain 1 and 2 (circled respectively by yellow and green) and the corresponding growth history for the fixed bi-crystallography $\left(30^{\circ}, 10^{\circ}\right)$ and (a) $G=1900 \mathrm{~K} \mathrm{~m}^{-1}, \delta_{t r, 1}=280 \mu \mathrm{m}$, (b) $G=475 \mathrm{~K} \mathrm{~m}^{-1}, \delta_{t r, 1}=550$ and $\delta_{t r, 2}=$ $670 \mu \mathrm{m}$, (c) $G=237.5 \mathrm{~K} \mathrm{~m}^{-1}, \delta_{t r, 1}=830$ and $\delta_{t r, 2}=845 \mu \mathrm{m}$. Note that the $0.5 \mathrm{~mm}$ scale bar is shorter in (c) than in (a) and (b). 
The depth $\delta_{t r}$ is further characterized and shown in Fig. 10. Fig. 10a shows the depth of tertiary branching, $\delta_{t r}$, and the associated limits of stable primary spacing, $\Lambda^{(1)}$, for a well-oriented single columnar dendrite growing with $G=475$ and $1900 \mathrm{~K} \mathrm{~m}^{-1}$. For $G=475 \mathrm{~K} \mathrm{~m}^{-1}$ the lower spacing limit below which dendrite elimination occurs $\sim 130 \mu \mathrm{m}$, and the upper spacing limit beyond which tertiary branches stably grow $\sim 705 \mu \mathrm{m}$ are marked by red patches. The corresponding depth below the tip at which tertiary branches start is $\overline{\delta_{t r}}=638 \mu \mathrm{m}$. Similarly, at $G=1900 \mathrm{~K} \mathrm{~m}^{-1}$ the lower and the upper spacing limits marked by blue patches are $\sim 110$ and 440 $\mu \mathrm{m}$, respectively, and $\overline{\delta_{t r}}=337 \mu \mathrm{m}$. Fig. 10a reaffirms the smaller temperature gradient, tertiary branches start deeper within the GB region, where suitable undercooling condition leads to the growth of a stable tertiary branch. It also shows that for a smaller $G$, the range of stable spacing is wider by $\sim 1.75$ times.

It is worth noting that, from Fig. 9, one may naively expect that the growth advantage of the tertiary branches of the well-oriented grain could be due to the fact that they first appear at a different depth inside the mushy zone than the tertiary branches of the misoriented grain $\left(\delta_{t r, 2}>\right.$ $\left.\delta_{t r, 1}\right)$. However, our measurements of a few simulations under the same condition (for the purpose of statistical analysis) show that the relation $\delta_{t r, 2}>\delta_{t r, 1}$ in the GL dominance regime does not always hold. In fact, both $\delta_{t r, 1}$ and $\delta_{t r, 2}$ tend to have comparable values within the statistical uncertainty of our measurements extracted from stochastic simulations. Fig. 10b shows the depth from the most advanced tip of tertiary branching events starting on either grain 1 or 2 at the diverging GB as a function of $G$ at bi-crystallographies $\left(20^{\circ}, 10^{\circ}\right),\left(30^{\circ}, 10^{\circ}\right)$, and $\left(40^{\circ}, 10^{\circ}\right) . \delta_{t r}$ for the case of a well-oriented single dendrite at the upper critical spacing is also plotted. Both the 
single grain and bi-crystal exhibit nearly the same values of $\delta_{t r}$ and follow the scaling law $\delta_{t r} \propto$ $G^{-0.5}$

(a)

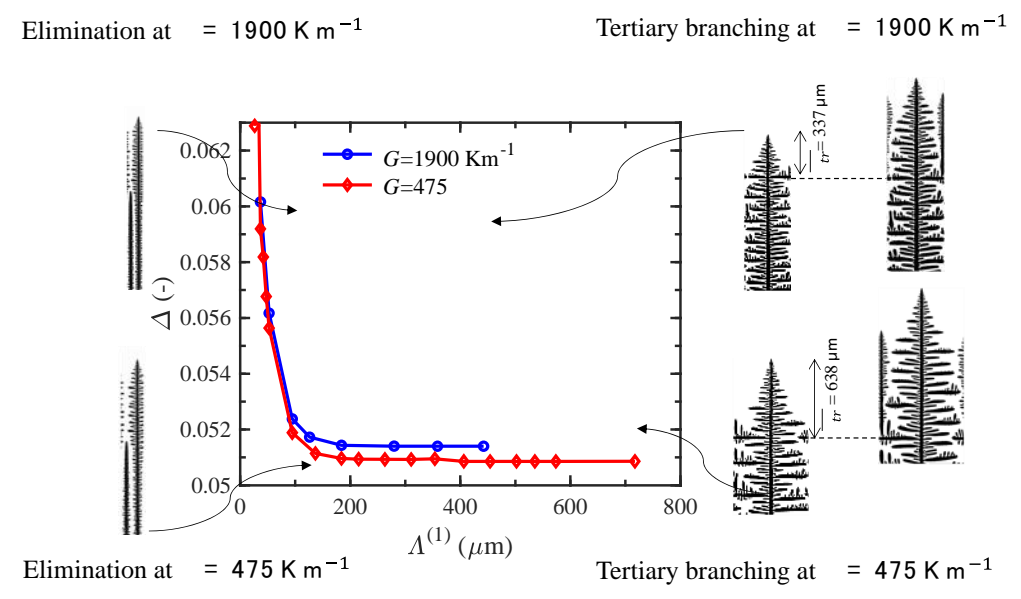

(b)

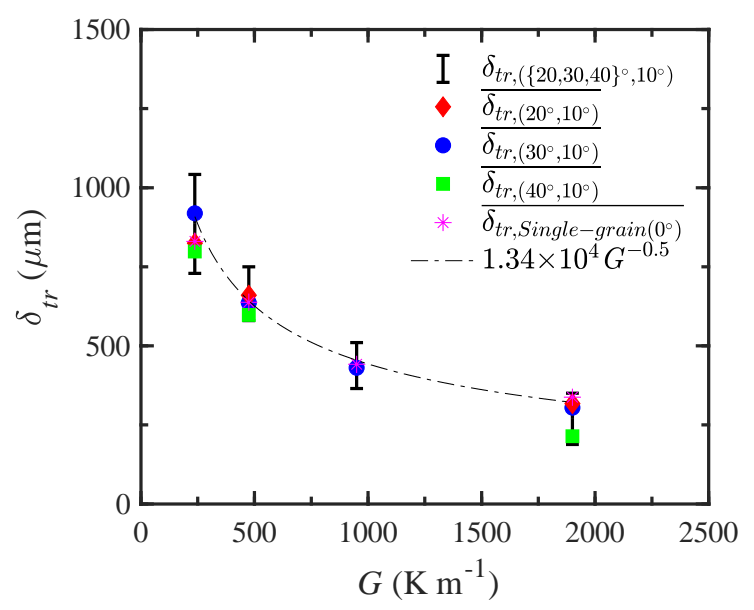

Fig. 10. (a) Limits of stable primary spacing, $\Lambda^{(1)}$, and depth, $\delta_{t r}$, of tertiary branching events for a welloriented $\left(0^{\circ}\right)$ dendritic grain with $G=475$ and $1900 \mathrm{~K} \mathrm{~m}^{-1}$. (b) $\delta_{t r}$ for both grain 1 and grain 2 as a function of $G$ for different bi-crystallographies $\left(20^{\circ}, 10^{\circ}\right),\left(30^{\circ}, 10^{\circ}\right)$, and $\left(40^{\circ}, 10^{\circ}\right)$, as well as the single $0^{\circ}$ grain. The black dashdotted line following the power law $\delta_{t r} \propto G^{-0.5}$ is fit to the average value of $\delta_{t r}$ from all bicrystallographies and the $0^{\circ}$ grain. The range of values shown by the black error bars include the three bicrystallographies $\left(20^{\circ}, 10^{\circ}\right),\left(30^{\circ}, 10^{\circ}\right)$, and $\left(40^{\circ}, 10^{\circ}\right)$.

Fig. 11 shows the range and average value of primary arm spacing resulting from tertiary branching on grain 1 (Fig. 11a) and grain 2 (Fig. 11b) at the diverging GB for $\left(20^{\circ}, 10^{\circ}\right.$ ), 
$\left(30^{\circ}, 10^{\circ}\right)$, and $\left(40^{\circ}, 10^{\circ}\right)$. Fig. 11 a shows that at smaller temperature gradients, $G=237.5$ and $475 \mathrm{~K} \mathrm{~m}^{-1}$, tertiary branches can select a wider range of stable spacing (presented as black error bars) with a lower limit even smaller than the spacing at $G=1900 \mathrm{~K} \mathrm{~m}^{-1}$. A similar trend was observed for the stable primary spacing range of a single dendrite in Fig. 10a. On the other hand, for $G=1900 \mathrm{~K} \mathrm{~m}^{-1}$ tertiary branches grow at a consistent spacing close to its average value and regularly block any chance of growth of tertiary branches on grain 2 . The black dash-dotted line following the power law $\Lambda_{1}^{(1)} \propto G^{-0.5}$ is fitted to the average value of $\Lambda_{1}^{(1)}$ from $\left(20^{\circ}, 10^{\circ}\right)$ and $\left(30^{\circ}, 10^{\circ}\right)$ configurations. This scaling law agrees with the correlation observed in previous studies $[17,29]$. Our measurements showed a wider range of $\Lambda_{1}^{(1)}$ variation for the $\left(30^{\circ}, 10^{\circ}\right)$ compared to the $\left(20^{\circ}, 10^{\circ}\right)$ configuration. Here, for clarity of the graph in Fig. 11a, a single error bar, and power law fit that includes both configurations $\left(20^{\circ}, 10^{\circ}\right)$, and $\left(30^{\circ}, 10^{\circ}\right)$ is plotted because both configurations follow similar variation as a function of $G$, i.e., $\propto G^{-0.5}$. The $\left(40^{\circ}, 10^{\circ}\right)$ configuration that exhibits a degenerate microstructure is excluded in the $\Lambda_{1}^{(1)} \propto G^{-0.5}$ fit. Similarly, in Fig. 11b, the $\Lambda_{2}^{(1)} \propto G^{-0.5}$ fit applies to the primary arm spacing from tertiary branching on grain 2 .

Some observations are made regarding the dependence of $\Lambda_{1 / 2}^{(1)}$ on crystal angle $\alpha$. From Fig. 11a and b, it is seen that this dependence becomes pronounced at smaller $G=237.5 \mathrm{~K} \mathrm{~m}^{-1}$. As $\alpha$ increases from $10^{\circ}$ in grain 2 (Fig. $11 \mathrm{~b}$ is related to grain 2) to $20^{\circ}$ and $30^{\circ}$ in grain 1 (Fig. 11a is related to the grain 1), $\overline{\Lambda_{1 / 2}^{(1)}}$ increases from 480 to 490 and $630 \mu \mathrm{m}$, respectively. Fig. 11c shows this variation of $\overline{\Lambda_{1 / 2}^{(1)}}$ with respect to $\alpha$ for $G=237.5 \mathrm{~K} \mathrm{~m}^{-1}$. This increasing trend does not hold for $40^{\circ}$ where degenerate structures with $\Lambda^{(1)} \approx \Lambda^{(2)}$ form; hence it is excluded from Fig. 
11c. Previous studies on the effect of crystal axis angle on primary spacing selection [17] predict a dependence of spacing on $\alpha$ using the expression:

$$
\Lambda^{(1)} \propto \Delta T_{0}^{a} V^{-b} G^{-c}\left[1+d\left((\cos \alpha)^{-e}-1\right)\right]
$$

where $\Delta T_{0}$ is the alloy freezing range and constant values of $a=b=0.25$ and $c=0.5, d \approx 0.15$ and $\mathrm{e} \approx 8$ [17] have been suggested for the references' condition (SCN-3.61 wt.\% Ace., $V=$ $[20-1000] \mu \mathrm{m} \mathrm{s}^{-1}$, and $\left.G=[100-10000] \mathrm{K} \mathrm{m}^{-1}\right)$. In Fig. 11c the fit to relation $\Lambda^{(1)}=$ $L F(\alpha) G^{-c}$, with $F(\alpha)=\left[1+0.15\left((\cos \alpha)^{-8}-1\right)\right]$ and $L=7.18 \mu \mathrm{m}^{0.5} \mathrm{~K}^{0.5}$ shows a good agreement between our results in the range $\alpha=10^{\circ}$ to $30^{\circ}$ and the expression proposed in [17].

(a)

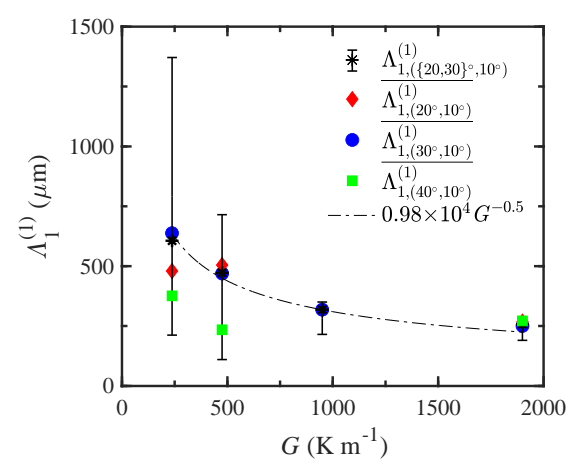

(b)

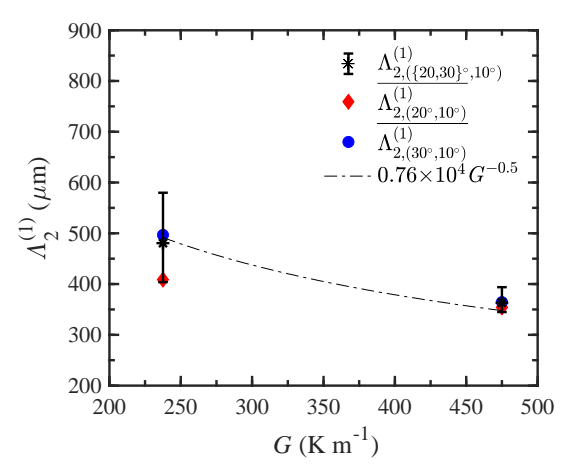

(c)

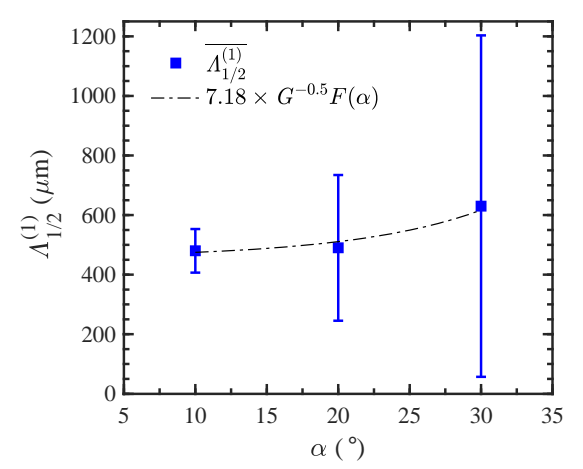

Fig. 11. Primary arm spacing resulting from tertiary branching of (a) grain 1, and (b) grain 2 , at the diverging GB as a function of $G$ for different bi-crystallographies $\left(20^{\circ}, 10^{\circ}\right),\left(30^{\circ}, 10^{\circ}\right)$, and $\left(40^{\circ}, 10^{\circ}\right)$. The black dashdotted line following the power law $\Lambda_{1 / 2}^{(1)} \propto G^{-0.5}$ is fit to the average value of $\Lambda_{1 / 2}^{(1)}$ from combination of $\left(20^{\circ}, 10^{\circ}\right)$, and $\left(30^{\circ}, 10^{\circ}\right)$ bi-crystallographies presented by black stars. The range of values shown by the black error bars include both $\left(20^{\circ}, 10^{\circ}\right),\left(30^{\circ}, 10^{\circ}\right)$ bi-crystallographies. (c) Variation of average primary arm spacing $\Lambda_{1 / 2}^{(1)}$ with respect to crystal angle $\alpha$ at $G=237.5 \mathrm{~K} \mathrm{~m}^{-1}$. 
The grain envelopes and the secondary arm spacings at the instances that a stable tertiary branch starts on its corresponding grain as introduced in Fig. 1c are characterized in Fig. 12. For the grain envelope profile, the length of the secondary arms, $L_{1 / 2, i}$, versus their distance from the tip of the trunk, $Z_{1 / 2, i}$, for grain 1 and grain 2 at various $G=237.5,475,950$, and $1900 \mathrm{~K} \mathrm{~m}^{-1}$ and fixed configuration $\left(30^{\circ}, 10^{\circ}\right)$ are plotted in Fig. 12a. The curve line connecting the active branches ( $i=2$ to 7 ) reflects the shape of the grain envelope. Previous experimental [38-42] and numerical $[33,43,44]$ studies show that the length of the active sidebranches, $L$, follow a simple power law with the distance from the primary dendrite tip, $Z$, as:

$$
\frac{L}{\rho_{\text {tip }}}=A\left(\frac{|Z|}{\rho_{\text {tip }}}\right)^{B}
$$

with $\rho_{\text {tip }}$ being the primary arm tip radius. Fig. 12 a shows that the active tips form concave fronts, all being enclosed approximately between power law fits $4.87 \times 10^{-4} Z^{2.24}$ and $4.36 \times 10^{-4} Z^{2.04}$. Fig. $12 \mathrm{~b}$ shows, at fixed bi-crystallography $\left(30^{\circ}, 10^{\circ}\right)$, as the distance from the tip increases (i.e., $i$ varies from 7 to 2 ), the average spacing between active secondary branches, $\Lambda_{2, i \& i+1}^{(2)}$, and their corresponding length, $L_{1 / 2, i}$, increases. Here, $\Lambda_{1 / 2, t r}^{(2)}$ reached approximately $5.7 l_{D}<\Lambda_{1 / 2, t r}^{(2)}<$ $31 l_{D}$ and the associated length of the branch reached $26.5 l_{D}<L_{1 / 2, i=2}<106 l_{D}$ as $G$ varied from 1900 to $237.5 \mathrm{~K} \mathrm{~m}^{-1}$. The fact that $\Lambda_{1 / 2, t r}^{(2)}>5 l_{D}$ indicates the active secondary branch preceding tertiary branching resides in a region with negligible (intra-grain) solutal interaction with other active branches from the same grain. However, it may or may not be in (inter-grain) solutal interaction with the active branches of the neighboring grain. Fig. 12a shows, the length of the active sidebranch at $i=2$ approach the length of its corresponding primary trunk, i.e., $L_{1 / 2, i=2} \approx$ 
$Z_{1 / 2, i=2}$ where it gets constrained by the neighboring grain and starts giving rise to a stable tertiary branch.

In Fig. 12b, the closest branch to the tip of the trunk (i.e., branch with index $i=7$ ) has a distance of approximately $Z_{1 / 2, i=7}>9 l_{D}$ from the tip of the trunk, length of $L_{1 / 2, i=7}>3.5 l_{D}$, and spacing of $\Lambda_{1 / 2, i=6 \& 7}^{(2)}>2 l_{D}$. At this stage, due to the diffusive interaction among branches, some sidebranches have been eliminated (i.e., decelerated and stopped). As a result of the progressive elimination of some sidebranches, the average secondary spacing, $\Lambda^{(2)}$, between surviving (active) branches has increased. To describe this progressive elimination, a linear relationship between this spacing $\Lambda^{(2)}$ and the corresponding sidebranch length, $L$, has been proposed theoretically [45-47], experimentally [48], and numerically [49], [50]. Fig. 12b validates this linear relation as $\Lambda_{1 / 2, i \& i+1}^{(2)}=b\left(L_{1 / 2, i}+L_{1 / 2, i+1}\right) / 2$ with $0<b<1$.

Competition at the diverging GB occurs through impingement of active secondary branches from the two grains. Active secondary arms that give rise to a stable tertiary branch can be of major importance because they feed and maintain the inter-grain occupancy during grain competition. Fig. 12c plots the active secondary arm spacing preceding tertiary branching, $\Lambda_{1 / 2, t r}^{(2)}$, versus the corresponding distance from the tip, $Z_{1 / 2, t r}=0.5\left(Z_{1 / 2, i=2}+Z_{1 / 2, i=3}\right)$, at nondegenerate configurations $\left(20^{\circ}, 10^{\circ}\right)$ and $\left(30^{\circ}, 10^{\circ}\right)$. A sublinear trend is observed, suggesting that both $\Lambda_{1 / 2, t r}^{(2)}$ and $Z_{1 / 2, t r}$ have similar variation as a function of $G . \Lambda_{1 / 2, t r}^{(2)}$ is further characterized in what follows.

(a) 

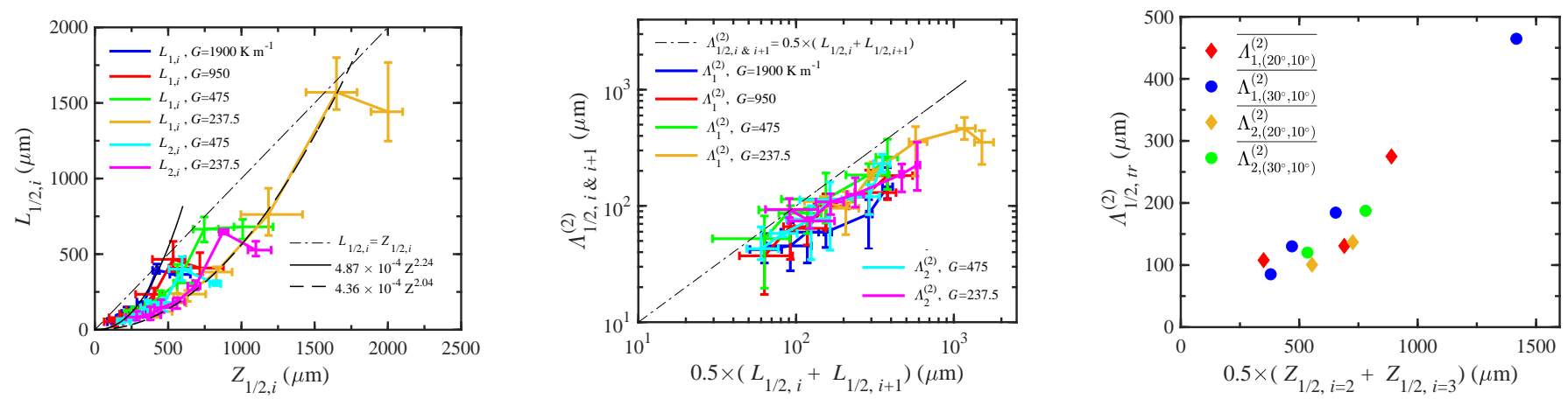

Fig. 12. (a) Grain envelope as measured by the length, $L_{1 / 2, i}$, of the secondary arms versus their distance from the tip of the trunk for grain $1, Z_{1, i}$, and grain $2, Z_{2, i}$, at various $G=237.5,475,950$, and $1900 \mathrm{~K} \mathrm{~m}^{-1}$, and fixed bi-crystallography $\left(30^{\circ}, 10^{\circ}\right), i=1$ to 7 are considered and active tips which form the grain envelope correspond to $i=2$ to 7 . (b) Secondary arm spacing on grain $1, \Lambda_{1, i \& i+1}^{(2)}, i=1$ to 6 , and grain $2, \Lambda_{2, i}^{(2)} \& i+1$, versus length of the secondary branches at various $G=237.5,475,950$, and $1900 \mathrm{~K} \mathrm{~m}^{-1}$, and fixed bicrystallography $\left(30^{\circ}, 10^{\circ}\right)$. (c) Active secondary arm spacing preceding tertiary branching, $\Lambda_{1 / 2, t r}^{(2)}$, versus $Z_{1 / 2, \text { tr }}=0.5\left(Z_{1 / 2, i=2}+Z_{1 / 2, i=3}\right)$ at $\left(20^{\circ}, 10^{\circ}\right)$ and $\left(30^{\circ}, 10^{\circ}\right)$ bi-crystallography.

Fig. 13a plots the active secondary arm spacing preceding tertiary branching, $\Lambda_{1 / 2, t r}^{(2)}$, as a function of $G$, at non-degenerate configurations $\left(20^{\circ}, 10^{\circ}\right)$ and $\left(30^{\circ}, 10^{\circ}\right)$. For comparison, the corresponding value of $\delta_{S t}$ is shown in the same plot. The secondary arm spacing increases as $G$ decreases. For grain 2 , which is the most favorably oriented grain, this variation can be estimated by $\Lambda_{2, t r}^{(2)} \propto G^{-0.5}$. However, grain 1 , which is the unfavorably oriented grain, shows different behavior, and a best fit to a power between -0.5 to -1 . It also shows that the secondary arm spacings on grain $2, \Lambda_{2, t r}^{(2)}$, are finer than the corresponding secondary arm spacings on grain $1, \Lambda_{1, t r}^{(2)}$. In the FOG limit, the active secondary arm spacing preceding tertiary branching, $\Lambda_{1 / 2, t r}^{(2)}$, can only be defined for the unfavorably oriented grain (i.e., grain $1, \Lambda_{1, t r}^{(2)}$ ) as it is the only grain that grows stable tertiary branching. However, in the GL dominance limit, the parameter $\Lambda_{1 / 2, t r}^{(2)}$ is defined for both grains and the finer spacing is related to the favorably oriented grain (i.e., grain 2). The effect 
of crystal orientation on secondary arm spacing is pronounced at smaller $G$, e.g., $G=237.5-$ $475 \mathrm{~K} \mathrm{~m}^{-1}$, where it is shown that the grain with larger misorientation has larger secondary arm spacing. A similar observation was made regarding the dependence of the primary arm spacing on the crystal angle.

Fig. 13a-b shows that in the GL dominance regime, i.e., $G=237.5-475 \mathrm{~K} \mathrm{~m}^{-1}$, at nondegenerate bi-crystallography, both $\delta_{S t}$ and $\Lambda_{2, t r}^{(2)}$ are of similar order, while it is not always the case when comparing $\Lambda_{1, t r}^{(2)}$ with $\delta_{S t}$. Fig. $13 \mathrm{~b}$ shows that by comparing the variation of $\Lambda_{2, t r}^{(2)}$ and $\delta_{S t}$ as a function of $G, \Lambda_{2, t r}^{(2)}$ variation occurs at a slower rate, i.e., $\Lambda_{2, t r}^{(2)} / \delta_{S t}$ decreases as $G$ decreases. However, grain 1 does not maintain the same behavior over the entire range of $G$. This indicates that, in the GL dominance regime, $\Lambda_{2, t r}^{(2)}$ (relative to $\delta_{S t}$ ) is the controlling length scale in grain competition between the two columnar grains.

In the FOG dominance regime, i.e., $G=950-1900 \mathrm{~K} \mathrm{~m}^{-1}$, at non-degenerate bicrystallography, based on Fig. $13 \mathrm{a}, \Lambda_{1, t r}^{(2)}$ has a larger value compared to $\delta_{S t}$. Fig. $13 \mathrm{~b}$ shows that the $\Lambda_{1, t r}^{(2)}$ variation occurs at a slower rate, i.e., $\Lambda_{1, t r}^{(2)} / \delta_{S t}$ decreases as $G$ decreases. This means that in the FOG dominance regime, $\Lambda_{1, t r}^{(2)}$ relative to $\delta_{S t}$ controls the grain competition between the two columnar grains. In fact, the GB is formed through the impingement of the secondary arms. Hence, active secondary arm spacing is the length scale directly involved in the grain competition and forming the GB trajectory. Particularly, the active secondary branch that gives rise to a stable tertiary branch, $\Lambda_{1 / 2, t r}^{(2)}$, feeds and maintains the inter-grain occupancy in favor of its parent grain, and subsequently defines the GB angle. 
By a similar analogy, in Fig. 13c, at degenerate bi-crystallography $\left(40^{\circ}, 10^{\circ}\right)$ in which $\Lambda_{1}^{(1)} \sim \Lambda_{1}^{(2)}$, variation of $\Lambda_{1}^{(1 / 2)} / \delta_{S t}$ with respect to $G$ is plotted. Fig. $13 \mathrm{c}$ shows that $\Lambda_{1}^{(1 / 2)}$ is greater or almost equal to $\delta_{S t}$ and its variation with respect to $G$ occurs at a slower rate than $\delta_{S t}$ variation with $G\left(\Lambda_{1}^{(1 / 2)} / \delta_{S t}\right.$ decreases as $G$ decreases). This implies that for a degenerate bi-crystallography, the value of $\Lambda_{1}^{(1 / 2)}$ relative to $\delta_{S t}$ controls the grain competition between the two columnar grains. At degenerate bi-crystallography, $\Lambda_{1}^{(1 / 2)}$ is the length scale that is physically involved in the formation of the GB trajectory. The aforementioned analyses made in recognizing the controlling length scale at each dominant regime and bi-crystallography provide the basis in bridging the PF and CA simulations of grain competition in the following section.

(a)

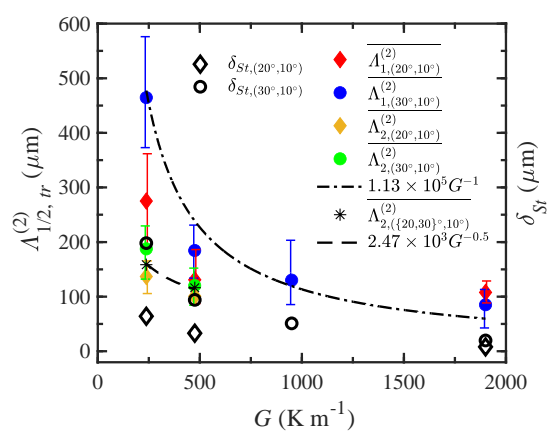

(b)

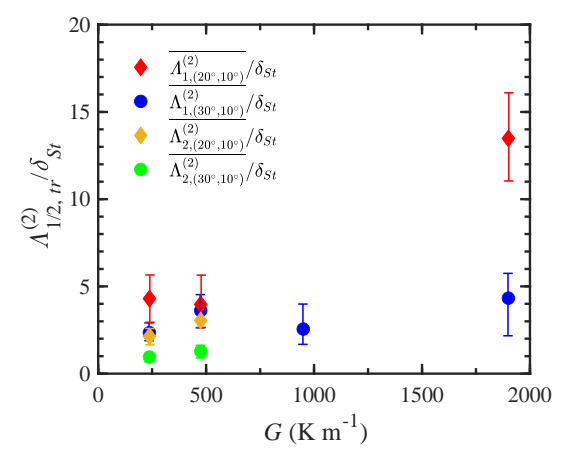

(c)

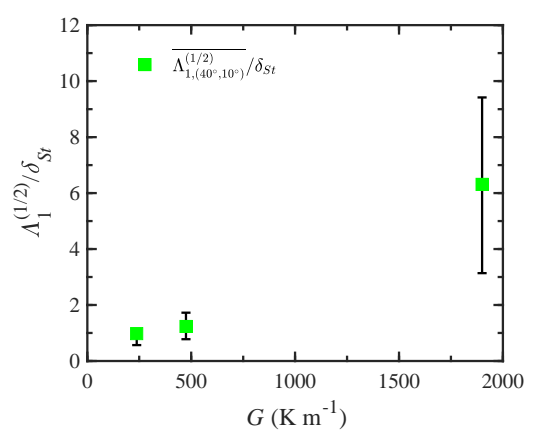

Fig. 13. (a-b) Spacing of active secondary branches preceding tertiary branching, $\Lambda_{1 / 2, t r}^{(2)}$, and $\delta_{S t}$ as a function of $G$, (a) plotted individually, and (b) as a ratio, for the non-degenerate bi-crystallographies $\left(20^{\circ}, 10^{\circ}\right)$ and $\left(30^{\circ}, 10^{\circ}\right)$. The black dashdotted line following the power law $\propto G^{-0.5}$ is fit to the average value of $\Lambda_{2, t r}^{(2)}$ from the combination of $\left(20^{\circ}, 10^{\circ}\right)$ and $\left(30^{\circ}, 10^{\circ}\right)$ bi-crystallographies presented by black stars. The range of values are shown by the error bars. (c) Ratio of the primary/secondary arm spacing and $\delta_{S t}$ as a function of $G$ at the degenerate bi-crystallography $\left(40^{\circ}, 10^{\circ}\right)$. 


\subsubsection{Evolution of the GB angle using the CA method}

In the current study, we perform CA simulations under the same condition as PF, corresponding to Table 1, with domain setting schematized in Fig. 4. First, we evaluate the evolution of the diverging GB angle in response to the cell size selection. Fig. 14 shows the diverging GB orientation angle, $\theta_{D}$, as a function of the temperature gradient, $G$, using CA cell size ranging from 50 to $400 \mu \mathrm{m}$ for a fixed couple of orientations angles $\left(30^{\circ}, 10^{\circ}\right)$. A similar observation as in the

previous study by Pineau et al. [8] is made regarding the cell size dependency of the CA simulations; at constant $G$, GB orientation is FOG dominant and GL dominant in the limit of large and small cell size, respectively [8]. The figure also shows that the CA simulations are able to predict the overall transition from FOG to GL by decreasing $G$ observed in the PF simulations. On the other hand, the CA simulations demonstrate the possibility to define a relevant cell size to predict the GB angle in agreement with PF. 


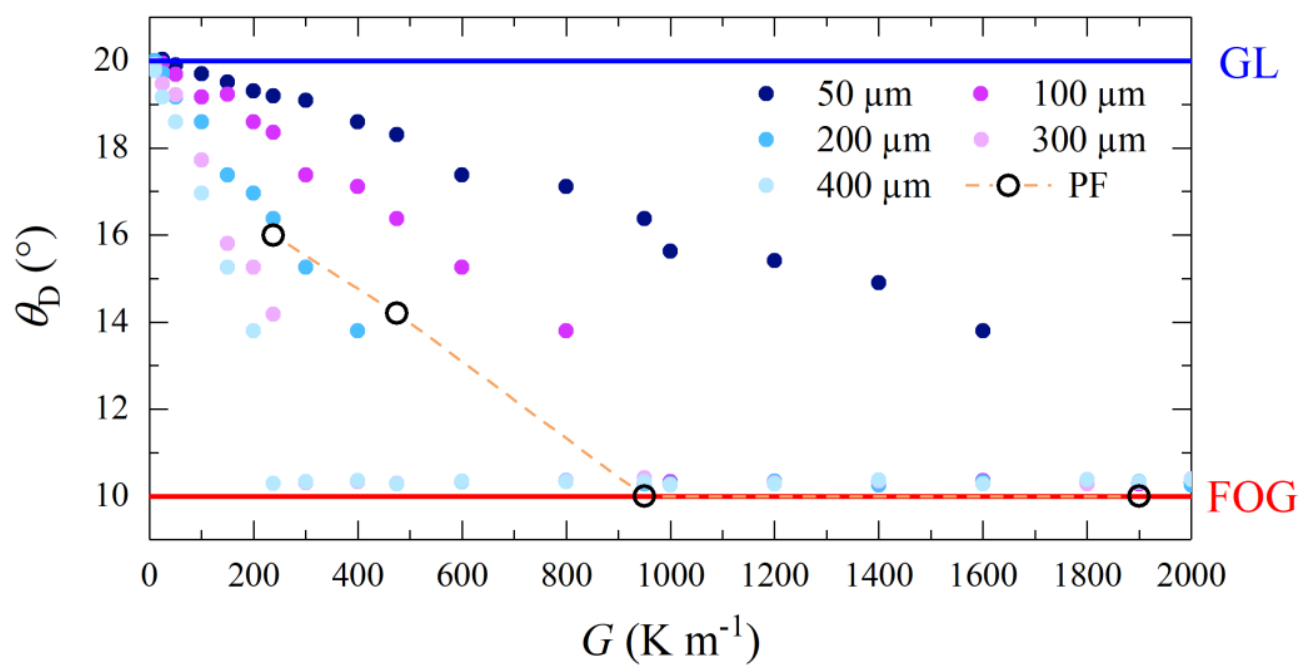

Fig. 14. CA simulations developed for fixed bi-crystallography $\left(30^{\circ}, 10^{\circ}\right)$. The evolution of divergent GB angle $\theta_{D}$ (dot symbols) is shown as a function of the imposed temperature gradient, $G$, for five values of the cell size, $l_{C A}=50$ to $400 \mu \mathrm{m}$. The GB angle associated with GL (resp. FOG) solution is shown with the horizontal blue (resp. red) plain line placed at position $\theta_{G L}=20^{\circ}$ (resp. $\theta_{F O G}=10^{\circ}$ ). Average results $\overline{\theta_{D}}$ of PF simulations for four investigated temperature gradients $\left(G=237.5,475,950\right.$, and $1900 \mathrm{~K} \mathrm{~m}^{-1}$ ) given in Fig. 6a are shown here with the orange dashed line and black circle symbols.

A precise estimation of the secondary arm spacing, $\Lambda^{(2)}$, value is difficult to obtain experimentally, as this value depends on the local composition, thermal condition, and crystal angle encountered at the groove location. Quantitative PF simulations provide an invaluable solution to isolate the estimation of this length scale and its evolution for application in calibrating CA as proposed hereafter. Based on the comprehensive analysis of the PF simulations obtained in section 3.1.3:

- At non-degenerate bi-crystallography, e.g., $\left(20^{\circ}, 10^{\circ}\right)$ and $\left(30^{\circ}, 10^{\circ}\right)$, in the GL dominance regime, the active secondary arm spacing preceding tertiary branching of the favorably oriented grain at the diverging GB (characterized in Fig. 13a.) is the controlling length scale in the grain competition. 
- At non-degenerate bi-crystallography, in the FOG dominance regime, the active secondary arm spacing preceding tertiary branching of the unfavorably oriented grain at the diverging GB (characterized in Fig. 13a.) is the controlling length scale in the grain competition.

- At degenerate microstructure with $\alpha_{1 / 2} \rightarrow 45^{\circ}$, e.g., $\left(40^{\circ}, 10^{\circ}\right)$ configuration, secondary and primary arms (characterized in Fig. 11a) are indistinguishable $\left(\Lambda^{(2)} \approx \Lambda^{(1)}\right)$ and they are the length scale that controls the grain competition.

Hence, as was conjectured in reference [23] and based on the tests performed on the CA simulations in the current and previous studies [8], we hypothesize that the CA method should be implemented with a choice of cell size as close as possible to the aforementioned length scales. In what follows, we further show that the aforementioned selection of the CA cell size will ensure that the current $\mathrm{CA}$ algorithm can best mimic the dynamics of the GB envelope profile at the finest scale of the branching that controls the GB; hence, the CA algorithm provides its best possible estimation of the GB angle in agreement with PF predictions.

CA simulations of interest correspond to the PF cases presented in Fig. 5. Three temperature gradients $G=237.5,475$, and $1900 \mathrm{~K} \mathrm{~m}^{-1}$ at $\left(20^{\circ}, 10^{\circ}\right),\left(30^{\circ}, 10^{\circ}\right)$, and $\left(40^{\circ}, 10^{\circ}\right)$ bicrystallography with angles reported in Fig. 6a have been investigated by CA simulations. All cases are performed for the diverging GB (i.e., $\alpha_{1}>\alpha_{2}$ ). Table 3 extracts the estimation of the proposed bridging length scale symbolized by $\bar{\Lambda}$ (from Figs. 11a and 13a, $\Lambda_{1 / 2, t r}^{(2)}$ ), and the associated average GB angle at the diverging $\mathrm{GB}, \overline{\theta_{D}}$, computed from PF simulations (from Fig. 6a, $\Lambda^{(1 / 2)}$ ) along with the corresponding $\delta_{s t}$ (from Fig. 3). 
Table 3. Summary of the PF simulation results for three bi-crystallographies $\left(\alpha_{1}, \alpha_{2}\right)$ and three temperature gradients, $G$, of interest. For non-degenerate configurations $\left(20^{\circ}, 10^{\circ}\right)$ and $\left(30^{\circ}, 10^{\circ}\right)$ the bridging length scale, $\bar{\Lambda}$, is extracted from Fig. 13a and for degenerate configuration $\left(40^{\circ}, 10^{\circ}\right)$ it is extracted from Fig. 11a. The average value of GB angles, $\overline{\theta_{D}}$, are extracted from Fig. 6a, and the corresponding $\delta_{S t}$ is taken from Fig. 3.

\begin{tabular}{|c|c|c|c|c|c|c|}
\hline Microstructure & $\begin{array}{c}\text { Angles } \\
\left(\alpha_{1}, \alpha_{2}\right) \\
\left(^{\circ}\right)\end{array}$ & $\begin{array}{l}\text { Dominant } \\
\text { regime } \\
\text { predicted } \\
\text { by PF }\end{array}$ & $\begin{array}{c}\text { Gradient } \\
G\left(\mathrm{~K} \mathrm{~m}^{-1}\right)\end{array}$ & $\delta_{S t}(\mu \mathrm{m})$ & $\begin{array}{l}\text { Bridging } \\
\text { length } \\
\text { scale } \bar{\Lambda} \\
\quad(\mu \mathrm{m})\end{array}$ & $\begin{array}{l}\text { Diverging } \\
\text { GB angle } \\
\overline{\theta_{D}}\left({ }^{\circ}\right)\end{array}$ \\
\hline \multirow[t]{3}{*}{ Non-degenerate } & \multirow{3}{*}{$\left(20^{\circ}, 10^{\circ}\right)$} & GL & 237.5 & 64 & 127 & 13.9 \\
\hline & & GL & 475 & 33 & 100 & 11.8 \\
\hline & & $\mathrm{FOG}$ & 1900 & 8 & 108 & 10 \\
\hline \multirow[t]{3}{*}{ Non-degenerate } & \multirow{3}{*}{$\left(30^{\circ}, 10^{\circ}\right)$} & GL & 237.5 & 198 & 187 & 16 \\
\hline & & GL & 475 & 94 & 120 & 14.2 \\
\hline & & FOG & 1900 & 20 & 85 & 10 \\
\hline \multirow[t]{3}{*}{ Degenerate } & \multirow{3}{*}{$\left(40^{\circ}, 10^{\circ}\right)$} & FOG & 237.5 & 385 & 496 & 10 \\
\hline & & FOG & 475 & 190 & 234 & 10 \\
\hline & & FOG & 1900 & 43 & 271 & 10 \\
\hline
\end{tabular}

Fig. 15 shows the evolution of the GB angle as a function of cell size for the 9 cases of interest predicted by CA simulations (purple dots). The vertical orange line is placed at the position of the best estimation of the relevant bridging length scale, $\bar{\Lambda}$, obtained from PF simulations. Fig. 15 shows a progressive and continuous transition of the GB angle from GL dominance (at small cell size) to FOG (at large cell size) regime, similar to the previous observations of Pineau et al. [8] and Fig. 14. With a cell size equal to $\bar{\Lambda}$, the CA simulations provide a GB angle, $\theta_{D}$, estimation in great agreement with the PF prediction.

For the largest temperature gradient, $G=1900 \mathrm{~K} \mathrm{~m}^{-1}$ (third column of Fig. 15), at nondegenerate bi-crystallography, $\left(20^{\circ}, 10^{\circ}\right)$ and $\left(30^{\circ}, 10^{\circ}\right)$, the $\mathrm{PF}$ simulation predicts $\theta_{D}$ equal to the FOG solution (i.e., $\theta_{D}=\alpha_{2}=10^{\circ}$ ). With a cell size equal to $\bar{\Lambda}$, the CA simulations lead to the FOG solution. Indeed, that is expected based on the conclusion made in the previous study [8], as 
the chosen cell size, $l_{C A}=\bar{\Lambda}$, is larger than the step $\delta_{S t}$ between the two grains. In this case, $l_{C A}=$ $\bar{\Lambda}$ specifically ensures that grain 1 is captured at the resolution of the secondary branching, while grain 2 is captured at the resolution of the primary arms.

For the degenerate bi-crystallography, $\left(40^{\circ}, 10^{\circ}\right)$, at all temperature gradients, $\mathrm{PF}$ simulations predict a $\bar{\Lambda}$ value larger than $\delta_{S t}$ (see Table 3 ) with FOG solution for GB angles, $\overline{\theta_{D}}$. At this configuration, the use of a cell size $l_{C A}$ equal to $\bar{\Lambda}$ also leads to the FOG solution in CA simulations. As demonstrated, CA solutions fall in an $l_{C A}$ domain where the GB angle is stabilized to the FOG angle. This CA behavior is also expected based on the previous study [8], as the chosen cell size, $l_{C A}=\bar{\Lambda}$, is larger than the step $\delta_{S t}$ between the two grains. In this case, $l_{C A}=\bar{\Lambda}$ warrants that grain 1 is captured at the resolution of the indistinct primary/secondary branching, while grain 2 is captured at the resolution of the primary arms.

In the GL dominance regime, i.e., cases of $G=237.5$ and $G=475 \mathrm{~K} \mathrm{~m}^{-1}$ at $\left(20^{\circ}, 10^{\circ}\right)$ and $\left(30^{\circ}, 10^{\circ}\right)$, slight differences up to the order of $\sim 0.5^{\circ}$ are present. Especially for the $\left(30^{\circ}, 10^{\circ}\right)$ configuration, this difference is very negligible. The observed deviations are partly due to the potential uncertainties in the estimation of $\Lambda^{(2)}$ and GB angles due to the number of simulation results. As it was earlier mentioned, for each case, 3 to 4 large-scale PF simulations are performed to account for the stochasticity inherent to the noise. Another source of deviation is the difference between the $\mathrm{GB}$ profile predicted by $\mathrm{CA}$ versus $\mathrm{PF}$, which originates from the inherent assumptions in the current CA model. The CA prediction of the GB envelope and its difference with $\mathrm{PF}$ is discussed in the following sections. 
(a)
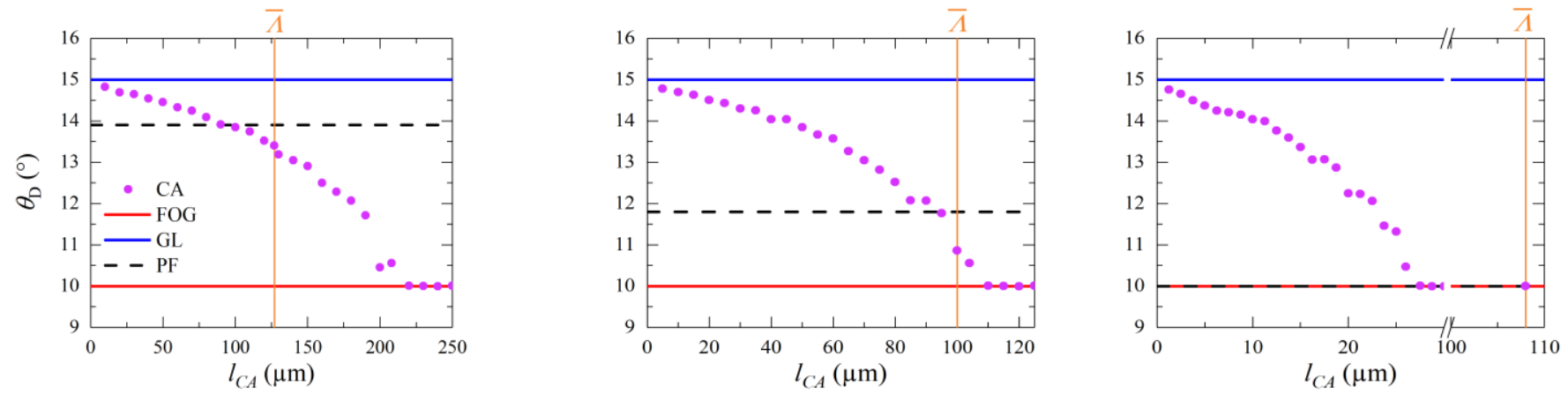

(b)
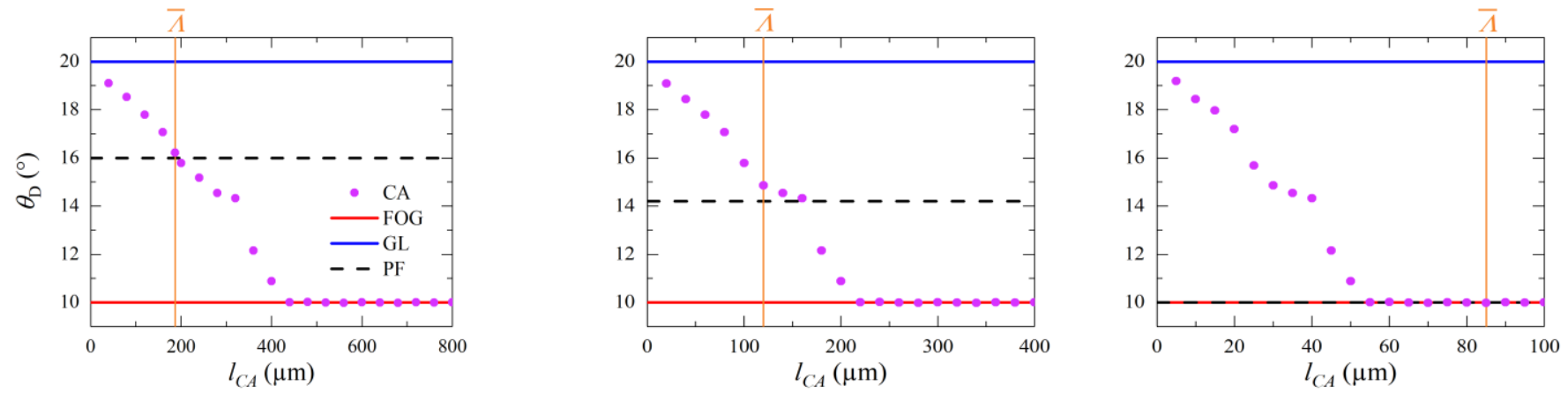

(c)
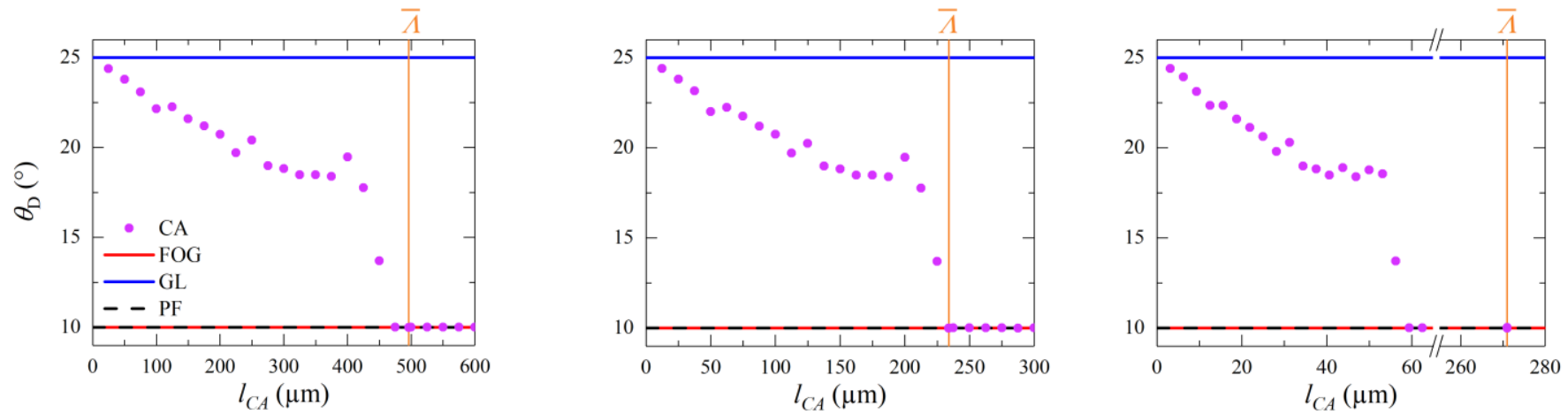

Fig. 15. Evolution of the diverging GB angle, $\theta_{D}$, in CA simulations (purple symbols). The crystal axis angle of grain $1, \alpha_{1}$, is equal to (a) $20^{\circ}$, (b) $30^{\circ}$ and (c) $40^{\circ}$ with the angle of grain $2 \alpha_{2}$ fixed at $10^{\circ}$. Three temperature gradients are investigated: $G=237.5 \mathrm{~K} \mathrm{~m}^{-1}$ (first column), $G=475 \mathrm{~K} \mathrm{~m}^{-1}$ (second column), and $G=1900 \mathrm{~K} \mathrm{~m}^{-1}$ (third column). The solution corresponding to GL (resp. FOG) prediction is illustrated as the blue (resp. red) horizontal line. The average GB angle from PF prediction, $\overline{\theta_{D}}$, (Fig. 6a) is shown with the horizontal dashed black line. The position of the relevant length scale, $\bar{\Lambda}$, bridging PF and CA (listed in Table 3 ) is illustrated with the vertical line. 
The GB regions developed from $\mathrm{CA}$ simulations for the nine cases of interest corresponding to Fig. 15, are drawn in Fig. 16. The cell size, $l_{C A}$, has been selected hereafter equal to the $\bar{\Lambda}$ value as estimated based on a set of PF simulations (Table 3 ). A steady state regime has been achieved in each case before capturing the GB region. The set of cells commonly located along the GB region reveal a specific angle with respect to the horizontal $x$-direction. This angle corresponds to the $\theta_{D}$ angle highlighted in Fig. 15 when $l_{C A}$ is equal to $\bar{\Lambda}$ (vertical orange line). We observe that for all cases, the cell size, $l_{C A}=\bar{\Lambda}$, is of the order of the step between grains, $\delta_{S t}$ (characterized in the Appendix), or larger. It leads to a rough GB region with no smooth transition between grains. This is because the $\theta_{D}$ angle is far from the absolute GL solution in all cases due to the current choices of solidification conditions imposed to the domain. However, a smoother transition with a more open GB region is observed at $\left(20^{\circ}, 10^{\circ}\right)$ (Fig. 16a) and $\left(30^{\circ}, 10^{\circ}\right)$ (Fig. 16b) when $G=237.5 \mathrm{~K} \mathrm{~m}^{-1}$ and $G=475 \mathrm{~K} \mathrm{~m}^{-1}$. These cases correspond to the $\theta_{D}$ governed by the GL dominance regime $\left(\theta_{F O G}<\theta_{D}<\theta_{G L}\right)$ (Fig. 15).

At $G=1900 \mathrm{~K} \mathrm{~m}^{-1}$, the GB region is rather consistent and small differences between the front advancements at the solid/liquid interface is observed regardless of the bi-crystallography; the GB domain is minimally open and a consistent GB position is observed corresponding to the FOG regime with $\theta_{D}=10^{\circ}$. Similarly, for the bi-crystallography, $\left(40^{\circ}, 10^{\circ}\right)$, we observe a consistent interface due to the weak competition between dendrite arms, seemingly restricted to the [10] axe closest to the horizontal direction in each grain. The GB region is closed with almost no liquid/solid interface. In this case, the best-oriented grain also imposes its orientation to the GB, leading to $\theta_{D}=10^{\circ}$ and the FOG regime. 
(a)
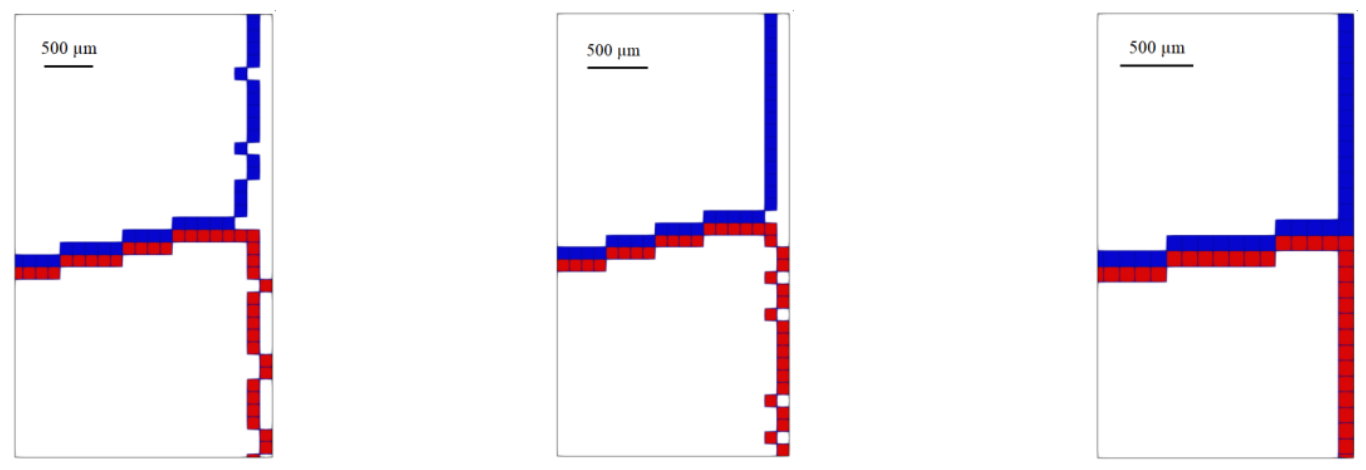

(b)
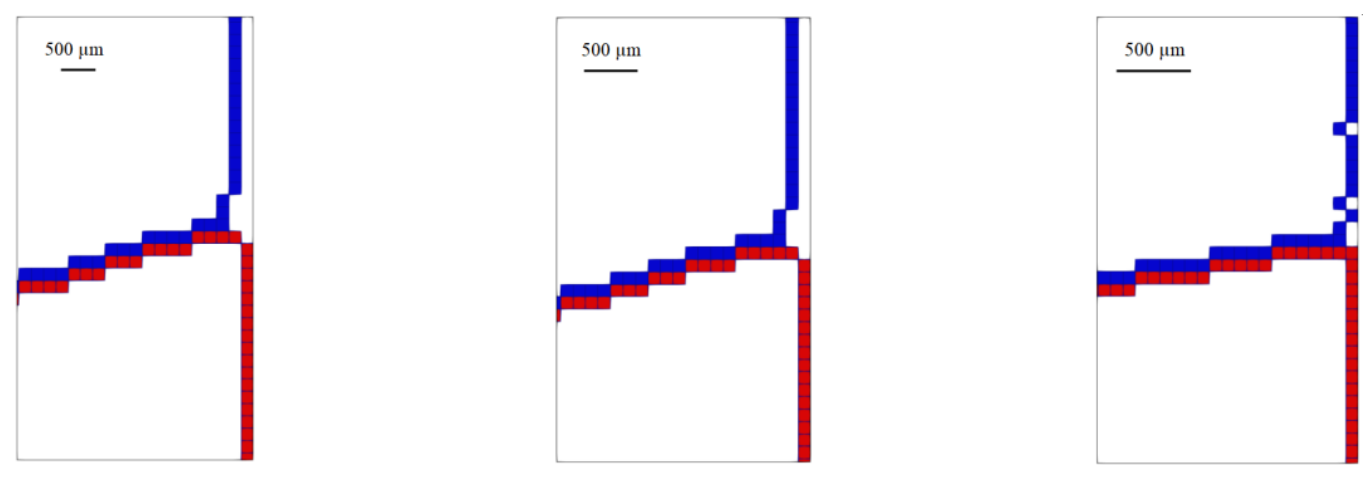

(c)
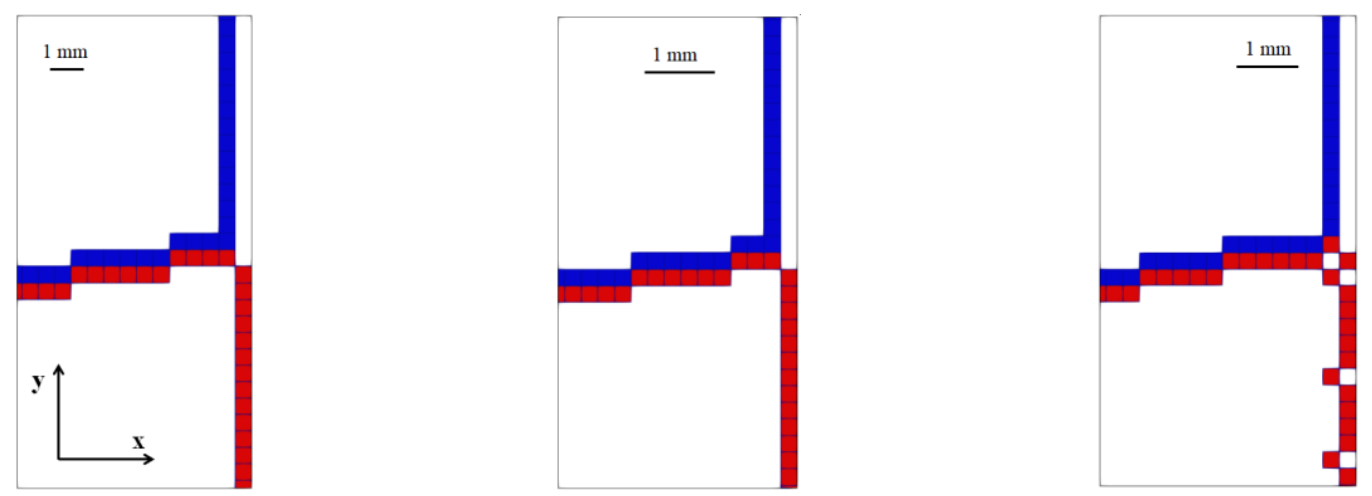

Fig. 16. Evolution of the GB shape in CA simulations (using $l_{C A}=\bar{\Lambda}$ listed in Table 3 ) at steady state. Orientation angle $\alpha_{1}$ (blue grain) is equal to (a) $20^{\circ}$, (b) $30^{\circ}$ and (c) $40^{\circ}$. Orientation angle $\alpha_{2}$ (red grain) is fixed at $10^{\circ}$. Three temperature gradients are investigated: $G=237.5 \mathrm{~K} \mathrm{~m}^{-1}$ (first column), $G=475 \mathrm{~K} \mathrm{~m}^{-1}$ (second column) and $G=1900 \mathrm{~K} \mathrm{~m}^{-1}$ (third column). 
Fig. 17 compares the GB profile obtained from $\mathrm{PF}$ and CA simulations (using $l_{C A}=\bar{\Lambda}$ ) at $\left(30^{\circ}, 10^{\circ}\right)$ and $G=237.5 \mathrm{~K} \mathrm{~m}^{-1}$ when the primary dendrite tips (in PF simulations) or the front profile (in CA simulations) far from the GB have reached steady growth. This state also corresponds to a stable GB groove in shape and temperature profile, only translating at the velocity $V / \cos \theta_{D}$ along the direction given by GB orientation $\theta_{D}$. Correlations are made shown by the blue (resp. red) solid lines that connect the tips of the growing CA polygonal shapes located at the GB for grain 1 (resp. grain 2) that define the grain envelopes. For better comparison, the corresponding correlation fit to the PF simulations in Fig. 12a shown by dashed lines are superimposed. In addition, in the background of the GB region, an instance of the PF GB structure is transparently superimposed. Note that since the fit to the PF simulations is done on the average profile from a few simulations, it does not entirely correspond to the one instance of the PF microstructure superimposed in Fig. 17. Correlations are both performed in the $\left(Z_{1 / 2, i}, L_{1 / 2, i}\right)$ reference frame. From PF simulations, due to the diffusive solutal interaction among secondary sidebranches, some sidebranches get eliminated so that the surviving (active) sidebranches form a concave envelope (dashed line). This concave front is highlighted by the exponents of the PF correlations equal to 2.04 (resp. 2.53) for grain 1 (resp. grain 2) which are higher than one.

On the contrary, in the CA method due to the absence of composition variations in the simulations, no solutal interaction between dendritic arms is accounted for. All the cells develop in the same solutal environment associated to the nominal acetone composition used to correlate the growth law (equation 3). As a result, the kinetics of the primary and secondary arms are the same, and their evolution is only controlled by the imposed temperature field. Simulation of the dynamics of the secondary branching at the diverging GB location is mimicked through the evolution of the growth envelopes using the proper choice of $l_{C A}$. Due to compromising the 
composition variations at the scale of the secondary arm spacing, the mechanism of sidebranch elimination due to the solute gradients is not captured. Consequently, a convex GB profile is usually observed when studying the development of a single grain envelope in a temperature gradient $[23,35,50,51]$, which is also why the sketch in Fig. 4 shows a convex profile.

The reason for the convex shape is explained in detail by considering the analytical analysis in reference [51] for a single grain. For the quadrant made by the secondary [10] branches and the primary/tertiary [01] branches of grain 1, the envelope shape is defined by the [01] tips as they correspond to a wider extension of the grain envelope compared to the [10] tips. The same is expected for grain 2 considering the quadrant made by secondary [10] branches and the primary/tertiary [01] branches, with wider extension of the [01] tips. In the present case, this convex grain shape is made visible by the solid blue line for the correlation associated with grain 1 and highlighted by the exponent in power law correlation equal to 0.79 (lower than 1 ). For grain 2, the front profile remained concave however follows an exponent of 1.40 exhibiting less curvature than the PF correlation. The reason for this concave front profile of grain 2 is attributed to the interaction of the grains at the GB, delaying the generation of [01] tertiary branching able to compete with $[\overline{1} 0]$ secondaries. The later situation is more similar to the PF prediction. The difference between PF and CA correlations of the GB front profile explains the persistence of slight difference between the GB angle predictions despite careful selection of $l_{C A}=\bar{\Lambda}$. Fig. 17 shows that geometrically relevant CA cell size deduced from PF simulations is necessary to properly mimic the dynamics of the secondary branching at the diverging GB and converge towards a correct estimation of the GB angle. 


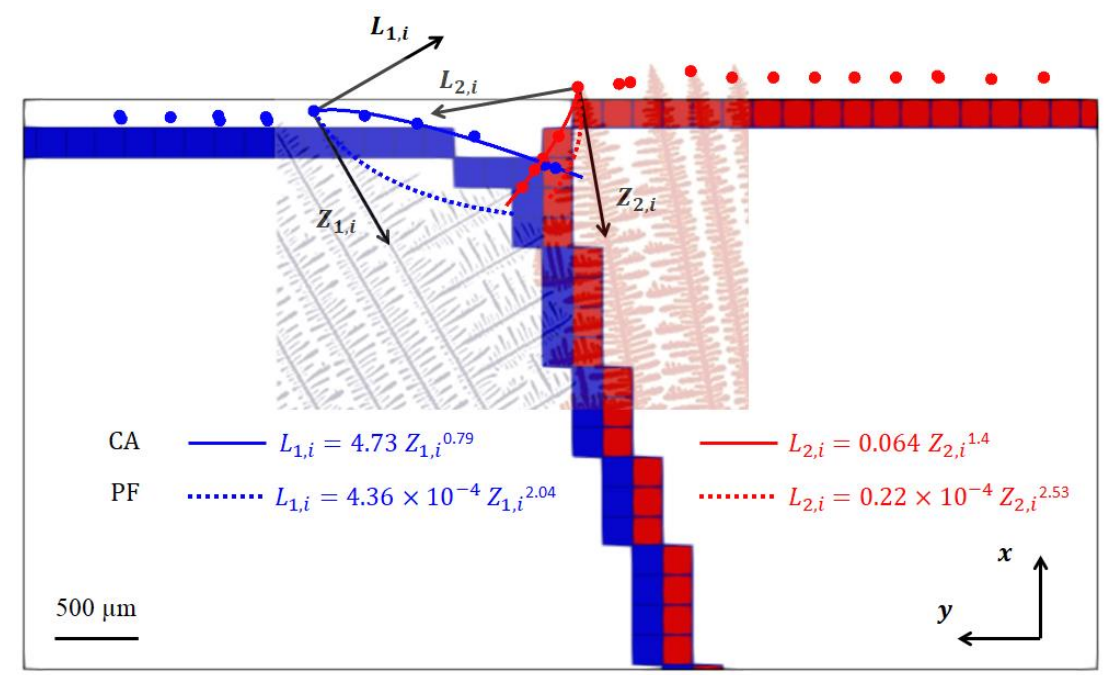

Fig. 17. Comparison of the GB shape predicted by $\mathrm{CA}$ and $\mathrm{PF}$ simulations for the $\left(30^{\circ}, 10^{\circ}\right)$ bicrystallography and $G=237.5 \mathrm{~K} \mathrm{~m}^{-1}$. Blue (resp. red) dots correspond to the positions of the most advanced $\langle 10\rangle$ tips of grain 1 (resp. grain 2) in CA simulations, defined from the growing polygonal shapes associated to the blue (resp. red) colored cells at the boundary with the liquid. Solid blue (resp. red) line represents the power law correlations on these tips located in the groove for grain 1 (resp. grain 2). Similarly, dashed blue (resp. red) line represents the power law correlation from PF simulations on the active tips in the same groove as reported in Fig. 12a.

The reproduction of the GB orientation at the diverging GBs by the CA method with the condition $l_{C A}=\bar{\Lambda}$ can be interpreted as follows.

- For the well-established dendritic regime under study (when the primary dendrite spacing is much larger than the solutal diffusion length), the GB orientation is the result of competition between active secondary and tertiary dendrite arms computed with branching length $l_{C A}=\bar{\Lambda}$. Thus, in the case of bi-crystalline activities of active secondary and tertiary branches, while a single branching length does not represent well the branching mechanisms of the favorably and unfavorably oriented grains, this does not prevent it from reaching agreement with the GB orientation predicted by PF. Indeed, this is made possible by properly mimicking the dynamics of the secondary branching (when needed) at the diverging GB location through an appropriate choice of $l_{C A}$. 
- Since the same branching length $\bar{\Lambda}$ is of the same order or larger than $\delta_{s t}$, the converging GB leads to FOG, in agreement with PF simulations.

- The region where solutal interaction is dominant, between the primary tip and the location $Z_{1 / 2, i=2}$ behind the tip, is not well represented by the current CA model. While PF systematically predicts a concave shape of the grain envelope, the CA growth algorithm tends to predict a more convex shape (see Fig. 17 grain 1).

\section{Summary, conclusions, and outlook}

We presented the results of an extensive study of the growth competition between columnar dendritic grains of an SCN-1.3 wt.\% Ace. alloy in a practically relevant, albeit to date computationally unexplored, growth regime where the primary dendrite spacing is much larger than the solutal diffusion length. This regime is characterized by the formation of hierarchical dendritic microstructures with fully developed primary, secondary, and tertiary branches that grow competitively to select in a non-trivial way the average GB orientation. Using quantitative PF simulations, we studied GB orientation selection as a function of the imposed temperature gradient, $G$, and GB bi-crystallography, for a fixed high pulling velocity that produces hierarchical dendritic microstructures. PF simulations at fixed non-degenerate bi-crystallography revealed a transition from the favorably-oriented-grain (FOG) to the geometrical limit (GL) with decreasing $G$. Simulations also provided a detailed quantitative characterization of the shape of the dendritic grain envelope and several internal microstructural length scales. This allowed us to identify for the first time, in conjunction with scale-bridging CA simulations, which length scale plays a dominant role in controlling the observed transition from FOG to GL. Among the several length scales that were investigated, including the primary and secondary dendrite arm spacings and the distance from the solidification front at which tertiary branching occurs, the secondary dendrite 
arm spacing preceding tertiary branching was found to play a dominant role in GB orientation selection. This was first suggested by the finding in PF simulations that this length scale, $\Lambda_{i, t r}^{(2)}$ (for grain $i=1$ and $i=2$ in the FOG- and GL-dominated regimes, respectively), has a different dependence on the temperature gradient $G$ than the steady distance between the fronts of the two grains, $\delta_{S t} \propto G^{-1}$, which is the only length scale present in CA simulations other than the cell size. This was then confirmed by CA simulations, further summarized below, which showed that the transition from FOG to GL with decreasing $G$ could be quantitatively reproduced for nondegenerate bi-crystallographies by choosing the cell size equal to $\Lambda_{i, t r}^{(2)}$. The position along the temperature axis at which tertiary branching occurs in the diverging GB region was found to obey the scaling law $\delta_{t r} \propto G^{-0.5}$, which is similar to the well-known empirical scaling law for the primary spacing. This length scale, however, could not explain the transition from FOG to GL because $\delta_{t r}$ versus $G$ curves are essentially indistinguishable for grains with different misorientation over the entire range of $G$ studied here, which encompasses this transition.

Previous CA simulations in [8] considered $\delta_{S t}$ as the threshold length for interpreting the CA behavior with respect to the cell size, $l_{C A}$. The study [8] suggested that the grain boundary orientation computed by the CA method depends on $l_{C A}$, so that at constant $G$, as $\frac{\delta_{S t}}{l_{C A}}$ increases, the GB orientation transitions from FOG to GL. Thanks to the present new PF simulations, by including the influence of the temperature gradient, additional length scales could be identified that were earlier conjectured to play a role [23]. PF simulations showed that the secondary arm spacing preceding tertiary branching increases as $G$ decreases. More specifically, for nondegenerate bi-crystallography, PF simulations in the GL dominance regime show that the variation of the secondary arm spacing preceding tertiary branching of the favorably oriented grain, $\Lambda_{2, t r}^{(2)}$, 
as a function of $G$ occurs at a slower rate than the variation of $\delta_{S t}$, as a function of $G$. This indicates that, in that regime, $\Lambda_{2, t r}^{(2)}$ relative to $\delta_{S t}$ is the length scale that controls the grain competition and GB orientation selection. Similarly, we found that, for non-degenerate bi-crystallography in the FOG regime, the secondary arm spacing preceding tertiary branching of the unfavorably oriented grain, $\Lambda_{1, t r}^{(2)}$, relative to $\delta_{S t}$ is the controlling length scale. Additionally, at degenerate bicrystallography, the primary and secondary arms are indistinct $\left(\Lambda^{(2)} \approx \Lambda^{(1)}\right)$ and they are the length scale that control the grain competition.

The CA method can capture the correct physics given a physically correct $l_{C A}$ as input. To reproduce the correct physics from CA simulations, we performed tests and analyses of the GB behavior obtained with respect to the $l_{C A}$ to identify the CA-PF bridging length scale. Our analyses show that at a non-degenerate microstructure, the CA method should be implemented with a choice of $l_{C A}$ as close as possible to the active secondary arm spacing preceding tertiary branching of the unfavorably or favorably oriented grain whichever has the finest spacing or is indeed present at a diverging GB. In the GL dominance regime, this length scale belongs to the favorably oriented grain where it is present, $\Lambda_{2, t r}^{(2)}$, and is finer than the length scale associated with the unfavorably oriented grain. We performed comparison between the CA and PF grain envelopes formed at the GB. It showed, implementing that criterion for selecting a physically relevant $l_{C A}$ ensures that CA captures the controlling length scale at the finest physically important resolution. Hence, CA can mimic the dynamics of the secondary sidebranching which is the hypothesis for the construction of the mathematical GL. At a degenerate microstructure $\left(\alpha_{1 / 2} \rightarrow 45^{\circ}\right), \Lambda^{(1)}$ is the controlling length scale which is proposed as the input cell size in CA simulations to capture the degenerate branching mechanism of the favorably oriented grain at its physically relevant resolution. 
Because the CA method was first developed for the simulation of grain structures in large domains, it was coupled with heat flow, for instance, using the FE method. This FE solution applied to casting in other solidification processing predicts large variation of $G$ and $V$ that also correspond to large variations of microstructure length scales, including $\bar{\Lambda}$. Hence the cell size could be adjusted in a so-called CA simulation where the grain structure is coupled with the FE solution of heat flow. This is presently not the case in the literature, and an automatic adaptive strategy of the CA grid would be required. It is yet to be considered that the present study is only focused on the effect of $G$ while other variables are known to play a major role on the microstructure length scales, such as the liquid flow and macrosegregation. In principle, all these variables could modify $\bar{\Lambda}$ and additional PF studies would be required to extend the validity of the present bridging scale results.

\section{Acknowledgements}

This research was part of the project CETSOL (Columnar to Equiaxed SOLidification processes) supported by NASA (National Aeronautics and Space Administration) under award No. 80NSSC19K0135 and by ESA (European Space Agency) under contract AO_99_117 14313/01/NL/SH.

\section{References}

[1] W. Kurz, D.J. Fisher, Fundamentals of solidification., Trans Tech Publications, Aedermannsdorf:, 1984. https://northeastern.on.worldcat.org/oclc/830340100 (accessed June 9, 2021).

[2] J.A. Dantzig, M. Rappaz, Solidification, Google Books. (2009). https://books.google.com/books/about/Solidification.html?id=yBDWQAAACAAJ (accessed June 9, 2021). 
[3] J. Li, Z. Wang, Y. Wang, J. Wang, Phase-field study of competitive dendritic growth of converging grains during directional solidification, Acta Materialia. 60 (2012) 1478-1493. https://doi.org/10.1016/j.actamat.2011.11.037.

[4] D. Tourret, A. Karma, Growth competition of columnar dendritic grains: A phase-field study, Acta Materialia. 82 (2015) 64-83. https://doi.org/10.1016/j.actamat.2014.08.049.

[5] T. Takaki, M. Ohno, Y. Shibuta, S. Sakane, T. Shimokawabe, T. Aoki, Two-dimensional phase-field study of competitive grain growth during directional solidification of polycrystalline binary alloy, Journal of Crystal Growth. 442 (2016) 14-24. https://doi.org/10.1016/j.jcrysgro.2016.01.036.

[6] D. Tourret, Y. Song, A.J. Clarke, A. Karma, Grain growth competition during thin-sample directional solidification of dendritic microstructures: A phase-field study, Acta Materialia. 122 (2017) 220-235. https://doi.org/10.1016/j.actamat.2016.09.055.

[7] D. Walton, B. Chalmers, The Origin of the Preferred Orientation in the Columnar Zone of Ingots, Transactions of the Metallurgical Society of AIME, Vol. 215. (1959) 3-13.

[8] A. Pineau, G. Guillemot, D. Tourret, A. Karma, C.A. Gandin, Growth competition between columnar dendritic grains - Cellular automaton versus phase field modeling, Acta Materialia. 155 (2018) 286-301. https://doi.org/10.1016/j.actamat.2018.05.032.

[9] N. D’Souza, M.G. Ardakani, A. Wagner, B.A. Shollock, M. McLean, Morphological aspects of competitive grain growth during directional solidification of a nickel-base superalloy, CMSX4, Journal of Materials Science. 37 (2002) 481-487. https://doi.org/10.1023/A:1013753120867. 
[10] A. Wagner, B.A. Shollock, M. McLean, Grain structure development in directional solidification of nickel-base superalloys, Materials Science and Engineering A. 374 (2004) 270-279. https://doi.org/10.1016/j.msea.2004.03.017.

[11] Y.Z. Zhou, A. Volek, N.R. Green, Mechanism of competitive grain growth in directional solidification of a nickel-base superalloy, Acta Materialia. 56 (2008) 2631-2637. https://doi.org/10.1016/j.actamat.2008.02.022.

[12] H. Esaka, K. Shinozuka, M. Tamura, Analysis of single crystal casting process taking into account the shape of pigtail, Materials Science and Engineering A. 413-414 (2005) 151155. https://doi.org/10.1016/j.msea.2005.08.154.

[13] T. Takaki, S. Sakane, M. Ohno, Y. Shibuta, T. Aoki, C.A. Gandin, Competitive grain growth during directional solidification of a polycrystalline binary alloy: Three-dimensional large-scale phase-field study, $\quad$ Materialia. $1 \quad$ (2018) 104-113. https://doi.org/10.1016/j.mtla.2018.05.002.

[14] T. Carozzani, C.A. Gandin, H. Digonnet, M. Bellet, K. Zaidat, Y. Fautrelle, Direct simulation of a solidification benchmark experiment, Metallurgical and Materials Transactions A: Physical Metallurgy and Materials Science. 44 (2013) 873-887. https://doi.org/10.1007/s11661-012-1465-1.

[15] C.A. Gandin, M. Rappaz, D. West, B.L. Ada, Grain texture evolution during the columnar growth of dendritic alloys, Metallurgical and Materials Transactions A. 26 (1995) 15431551. https://doi.org/10.1007/BF02647605.

[16] H. Esaka, Dendritic Growth and Spacing in Succinonitrile-Aceton Alloy, (1986) 119. https://doi.org/10.5075/EPFL-THESIS-615. 
[17] C.A. Gandin, M. Eshelman, R. Trivedi, Orientation dependence of primary dendrite spacing, Metallurgical and Materials Transactions A: Physical Metallurgy and Materials Science. 27 (1996) 2727-2739. https://doi.org/10.1007/BF02652367.

[18] T. Takaki, M. Ohno, T. Shimokawabe, T. Aoki, Two-dimensional phase-field simulations of dendrite competitive growth during the directional solidification of a binary alloy $\begin{array}{lllll}\text { bicrystal, } & \text { Acta } & \text { Materialia. } & 81 & \text { (2014) }\end{array}$ https://doi.org/10.1016/j.actamat.2014.08.035.

[19] C. Guo, T. Takaki, S. Sakane, M. Ohno, Y. Shibuta, T. Mohri, Overgrowth behavior at converging grain boundaries during competitive grain growth: A two-dimensional phasefield study, International Journal of Heat and Mass Transfer. 160 (2020) 120196. https://doi.org/10.1016/j.ijheatmasstransfer.2020.120196.

[20] T. Takaki, T. Shimokawabe, M. Ohno, A. Yamanaka, T. Aoki, Unexpected selection of growing dendrites by very-large-scale phase-field simulation, Journal of Crystal Growth. 382 (2013) 21-25. https://doi.org/10.1016/j.jcrysgro.2013.07.028.

[21] B. Echebarria, R. Folch, A. Karma, M. Plapp, Quantitative phase-field model of alloy solidification, Physical Review E - Statistical Physics, Plasmas, Fluids, and Related Interdisciplinary Topics. 70 (2004) 22. https://doi.org/10.1103/PhysRevE.70.061604.

[22] K. Ji, A.M. Tabrizi, A. Karma, Isotropic finite-difference approximations for phase-field simulations of polycrystalline alloy solidification, In Preparation. (2021).

[23] C.A. Gandin, M. Rappaz, A coupled finite element-cellular automaton model for the prediction of dendritic grain structures in solidification processes, Acta Metallurgica Et Materialia. 42 (1994) 2233-2246. https://doi.org/10.1016/0956-7151(94)90302-6. 
[24] C.A. Gandin, J.L. Desbiolles, M. Rappaz, P. Thévoz, A three-dimensional cellular automaton-finite element model for the prediction of solidification grain structures, Metallurgical and Materials Transactions A: Physical Metallurgy and Materials Science. 30 (1999) 3153-3165. https://doi.org/10.1007/s11661-999-0226-2.

[25] A. Saad, C.A. Gandin, M. Bellet, N. Shevchenko, S. Eckert, Simulation of Channel Segregation During Directional Solidification of In—75 wt pct Ga. Qualitative Comparison with In Situ Observations, Metallurgical and Materials Transactions A: Physical Metallurgy and Materials Science. 46 (2015) 4886-4897. https://doi.org/10.1007/s11661-015-2963-8.

[26] D.R. Liu, N. Mangelinck-Noël, C.A. Gandin, G. Zimmermann, L. Sturz, H. Nguyen-Thi, B. Billia, Simulation of directional solidification of refined Al-7 wt.\% $\mathrm{Si}$ alloys Comparison with benchmark microgravity experiments, Acta Materialia. 93 (2015) 24-37. https://doi.org/10.1016/j.actamat.2015.03.058.

[27] A. Karma, W.J. Rappel, Quantitative phase-field modeling of dendritic growth in two and three dimensions, Physical Review E - Statistical Physics, Plasmas, Fluids, and Related Interdisciplinary Topics. 57 (1998) 4323-4349. https://doi.org/10.1103/PhysRevE.57.4323.

[28] A. Karma, Phase-field formulation for quantitative modeling of alloy solidification, $\begin{array}{lllll}\text { Physical } & \text { Review } & \text { Letters. } & 87 & \text { (2001) 115701-1-115701-4. }\end{array}$ https://doi.org/10.1103/PhysRevLett.87.115701.

[29] D. Tourret, A. Karma, Growth competition of columnar dendritic grains: A phase-field study, Acta Materialia. 82 (2015) 64-83. https://doi.org/10.1016/j.actamat.2014.08.049.

[30] A. Karma, W.-J. Rappel, Phase-field model of dendritic sidebranching with thermal noise, Physical Review E. 60 (1999) 3614. https://doi.org/10.1103/PhysRevE.60.3614. 
[31] J. Deschamps, M. Georgelin, A. Pocheau, Growth directions of microstructures in directional solidification of crystalline materials, Physical Review E - Statistical, Nonlinear, and Soft Matter Physics. 78 (2008) 011605. https://doi.org/10.1103/PhysRevE.78.011605.

[32] D. Tourret, A. Karma, Multiscale dendritic needle network model of alloy solidification, Acta Materialia. 61 (2013) 6474-6491. https://doi.org/10.1016/j.actamat.2013.07.026.

[33] D. Tourret, A. Karma, Three-dimensional dendritic needle network model for alloy solidification, Acta $\quad$ Materialia. $120 \quad$ (2016) 240-254. https://doi.org/10.1016/j.actamat.2016.08.041.

[34] M.A. Chopra, M.E. Glicksman, N.B. Singh, Dendritic solidification in binary alloys, Metallurgical Transactions. A, Physical Metallurgy and Materials Science. 19 A (1988) 3087-3096. https://doi.org/10.1007/BF02647736.

[35] K. Somboonsuk, J.T. Mason, R. Trivedi, INTERDENDRITIC SPACING: PART I. EXPERIMENTAL STUDIES., in: Metallurgical Transactions. A, Physical Metallurgy and Materials Science, Springer, 1984: pp. 967-975. https://doi.org/10.1007/bf02644688.

[36] C.A. Gandin, M. Rappaz, A 3D cellular automaton algorithm for the prediction of dendritic grain growth, Acta Materialia. 45 (1997) 2187-2195. https://doi.org/10.1016/S13596454(96)00303-5.

[37] W. Kurz, B. Giovanola, R. Trivedi, Theory of microstructural development during rapid solidification, Acta Metallurgica. 34 (1986) 823-830. https://doi.org/10.1016/00016160(86)90056-8.

[38] S.C. Huang, M.E. Glicksman, Overview 12: Fundamentals of dendritic solidification-I. Steady-state tip growth, Acta Metallurgica. 29 (1981) 701-715. https://doi.org/10.1016/0001-6160(81)90115-2. 
[39] E. Hürlimann, R. Trittibach, U. Bisang, J.H. Bilgram, Integral parameters of xenon $\begin{array}{llllll}\text { dendrites, } & \text { Physical } & \text { Review } & \text { A. } & 46 & \text { (1992) }\end{array}$ https://doi.org/10.1103/PhysRevA.46.6579.

[40] Q. Li, C. Beckermann, Scaling behavior of three-dimensional dendrites, Physical Review E - Statistical Physics, Plasmas, Fluids, and Related Interdisciplinary Topics. 57 (1998) 31763188. https://doi.org/10.1103/PhysRevE.57.3176.

[41] Q. Li, C. Beckermann, Evolution of the sidebranch structure in free dendritic growth, Acta Materialia. 47 (1999) 2345-2356. https://doi.org/10.1016/S1359-6454(99)00115-9.

[42] A.J. Melendez, C. Beckermann, Measurements of dendrite tip growth and sidebranching in succinonitrileacetone alloys, Journal of Crystal Growth. 340 (2012) 175-189. https://doi.org/10.1016/j.jcrysgro.2011.12.010.

[43] Y. Souhar, V.F. De Felice, C. Beckermann, H. Combeau, M. Založnik, Three-dimensional mesoscopic modeling of equiaxed dendritic solidification of a binary alloy, Computational Materials Science. 112 (2016) 304-317. https://doi.org/10.1016/j.commatsci.2015.10.028.

[44] H. Xing, X. Dong, J. Wang, K. Jin, Orientation Dependence of Columnar Dendritic Growth with Sidebranching Behaviors in Directional Solidification: Insights from Phase-Field Simulations, Metallurgical and Materials Transactions B: Process Metallurgy and Materials Processing Science. 49 (2018) 1547-1559. https://doi.org/10.1007/s11663-018-1265-0.

[45] E. Brener, D. Temkin, Noise-induced sidebranching in the three-dimensional nonaxisymmetric dendritic growth, Physical Review E. 51 (1995) 351-359. https://doi.org/10.1103/PhysRevE.51.351. 
[46] M.E. Cates, Diffusion-limited aggregation without branching in the continuum approximation, Physical Review A. $34 \quad$ (1986) 5007-5009. https://doi.org/10.1103/PhysRevA.34.5007.

[47] K. Kassner, Solutions to the mean-field equations of branchless diffusion-limited aggregation, Physical Review A. $42 \quad$ (1990) 3637-3640. https://doi.org/10.1103/PhysRevA.42.3637.

[48] K. Kishinawa, H. Honjo, H. Sakaguchi, Scale-invariant competitive growth of side branches in a dendritic crystal, Physical Review E - Statistical, Nonlinear, and Soft Matter Physics. 77 (2008) 030602. https://doi.org/10.1103/PhysRevE.77.030602.

[49] H. Sakaguchi, K. Kishinawa, K. Katsuki, H. Honjo, Simple model for competitive dynamics among dendritic sidebranches, Physical Review E - Statistical, Nonlinear, and Soft Matter Physics. 75 (2007) 021606. https://doi.org/10.1103/PhysRevE.75.021606.

[50] G. Rossi, Diffusion-limited aggregation without branching: A detailed analysis, Physical Review A. 35 (1987) 2246-2253. https://doi.org/10.1103/PhysRevA.35.2246.

[51] C.A. Gandin, R.J. Schaefer, M. Rappaz, Analytical and numerical predictions of dendritic grain envelopes, Acta Materialia. 44 (1996) 3339-3347. https://doi.org/10.1016/13596454(95)00433-5.

[52] M. Abramowitz, I.A. Stegun, Handbook of Mathematical Functions - (Tenth printing ed.), National Institute of Standards and Technology, 1972.

\section{Appendix:}

In the current study, the GB orientation during a sufficiently large solidification time from $\mathrm{CA}$ and PF simulations is discussed. It is therefore of interest to estimate the time required to 
achieve a steady state regime during concurrent growth of two grains as illustrated in Fig. 3. with relative position, $x_{i}$, of the two solidification fronts, $\delta=\left|x_{2}-x_{1}\right|$, as $\delta$ is stabilized and a $\delta_{S t}$ value is achieved. Similarly, we assume that GB angle, $\theta$, remains stable in this condition, and no further evolution is observed.

In the present CA approach, a power law relation for the estimation of dendrite growth velocity is used (equation 3). Hence, for each grain $i \in\{1,2\}$ (Fig. 3) we have:

$$
\begin{gathered}
v_{x_{i}}=\cos \left(\alpha_{i}\right) v_{\alpha_{i}} \\
\frac{\partial x_{i}}{\partial t}=\cos \left(\alpha_{i}\right) A \Delta T_{i}^{n}
\end{gathered}
$$

where $\Delta T_{i}=T_{L}-T_{i}$ is the undercooling at the front for grain $i$, and $T_{L}$ is the liquidus temperature of the alloy. A fixed temperature gradient, $G$, with isotherm velocity, $V$, is imposed which corresponds to a constant cooling rate condition. Accordingly, the temperature at position $x$ is defined with the following expression:

$$
T=T_{0}+G\left(\left(x-x_{0}\right)-V\left(t-t_{0}\right)\right)
$$

where $T_{0}$ is the temperature at the initial time on the left-hand side part of the simulation domain in Fig. 3, i.e., $T\left(x_{0}, t_{0}\right)=T_{0}$. The relation between solidification front position, $x_{i}$, and time $t$ is thereafter given as:

$$
\frac{\partial x_{i}}{\partial t}=\cos \left(\alpha_{i}\right) A\left(T_{L}-\left(T_{0}+G\left(\left(x_{i}-x_{0}\right)-V\left(t-t_{0}\right)\right)\right)\right)^{n}
$$

We assume that solidification begins at time $t_{0}=0 \mathrm{~s}$ on the left-hand side part of the domain of position $x_{0}=0 \mathrm{~m}$ without nucleation undercooling leading to $T_{0}=T_{L}$. Consequently, the time relation (A.4) is simplified as:

$$
\frac{\partial x_{i}}{\partial t}=\cos \left(\alpha_{i}\right) A G^{n}\left(V t-x_{i}\right)^{n}
$$


We may define the positive variable $u_{i}$ associated with the distance between liquidus line and solidification front positions for each grain $i$ as:

$$
u_{i}=V t-x_{i}
$$

The differential equation is written using this $u_{i}$ variable as:

$$
\frac{\partial u_{i}}{\partial t}=V-\cos \left(\alpha_{i}\right) A G^{n} u_{i}^{n}
$$

A temporal integration of the latter equation can be done leading to:

$$
t=\frac{u_{i}}{V}{ }_{2} F_{1}\left[1, \frac{1}{n}, 1+\frac{1}{n}, \gamma u_{i}^{n}\right]
$$

where the variable $\gamma$ is equal to $\left(A \cos \cos \left(\alpha_{i}\right) G^{n} / V\right)$ and ${ }_{2} F_{1}$ is the hypergeometric function [52]. It is, therefore, possible to compute, at a given time $t$, the $u_{i}$ length when solving numerically the equation A.9. The step between the solidification front position is also equal to the difference between $u_{i}$ value at the same time $\left(\delta(t)=\left|u_{2}(t)-u_{1}(t)\right|\right)$. As expected, it is possible to demonstrate that $\delta$ converges toward a stable value $\delta_{S t}$ when time $t$ evolves toward infinite. This stable difference between front positions is equal to:

$$
\delta_{S t}=\frac{1}{G}\left(\frac{V}{A}\right)^{\frac{1}{n}}\left|\frac{1}{\cos \left(\alpha_{1}\right)^{\frac{1}{n}}}-\frac{1}{\cos \left(\alpha_{2}\right)^{\frac{1}{n}}}\right|
$$

Fig. A.1a shows an application of formula A.9 for the time estimation of $\delta$ value for three cases of interest $\left(\alpha_{1} \in\left\{20^{\circ}, 30^{\circ}, 40^{\circ}\right\}\right.$ and $\left.\alpha_{2}=10^{\circ}\right)$ at $G=1900 \mathrm{~K} \mathrm{~m}^{-1}$. The growth velocity parameters $A$ and $n$ are equal to the values provided in equation 3 and the isotherm velocity is fixed to $86 \mu \mathrm{m} \mathrm{s}^{-1}$. The variables converge toward stable values demonstrating achievement of a steady state regime after around $25 \mathrm{~s}$. This duration also has a small dependence on the angle choice. The limits are respectively equal to $\delta_{S t, 20^{\circ}, 10^{\circ}}=11.3 \mu \mathrm{m}, \delta_{S t, 30^{\circ}, 10^{\circ}}=31.2 \mu \mathrm{m}$ and $\delta_{S t, 40^{\circ}, 10^{\circ}}=62 \mu \mathrm{m}$ for the investigated temperature gradients. 
Similarly, Fig. A.1b shows the time evolution of step length for the single couple $\left(30^{\circ}, 10^{\circ}\right)$ and three gradients of interest $237.5 \mathrm{~K} \mathrm{~m}^{-1}, 475 \mathrm{~K} \mathrm{~m}^{-1}$ and $1900 \mathrm{~K} \mathrm{~m}^{-1}$. Progressive evolution toward stable value $\delta_{S t}$ are observed with $250 \mu \mathrm{m}, 125 \mu \mathrm{m}$ and $31.2 \mu \mathrm{m}$. As shown in equation (A.10), steady values are inversely proportional to temperature gradients. Times required to achieve stabilization also appears proportional to the temperature gradient.

(a)

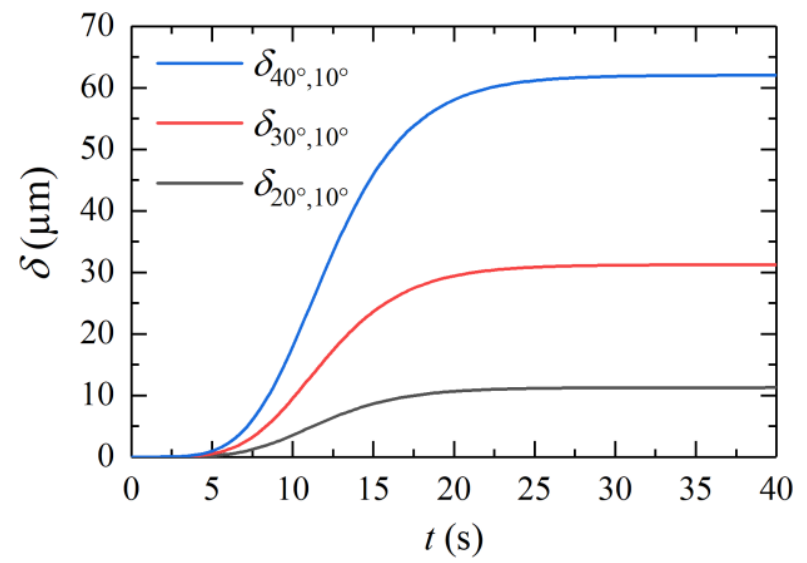

(b)

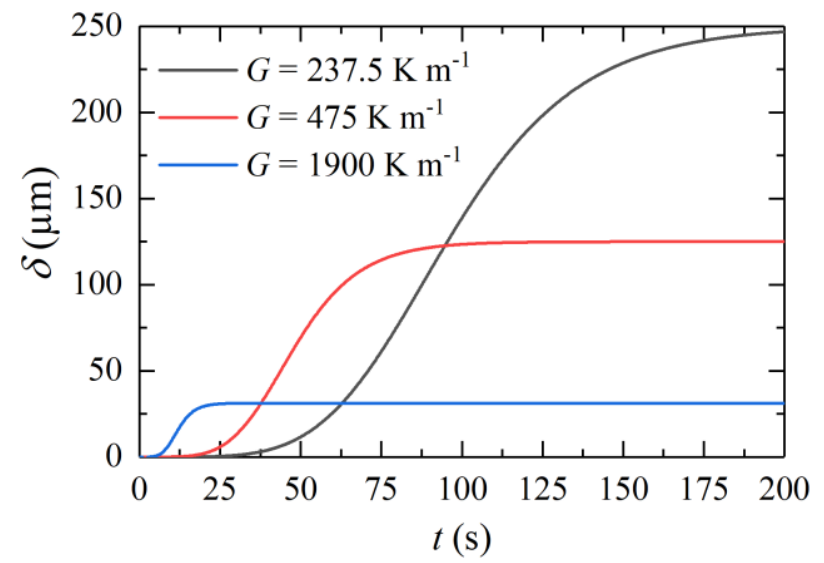

Fig A.1. Time evolution of the step, $\delta$, between two grains in growth competition for various cases of interest. a) The angle $\alpha_{1}$ is equal to $20^{\circ}, 30^{\circ}$ or $40^{\circ}$ and angle $\alpha_{2}$ is fixed to $10^{\circ}$ in all cases. The temperature gradient is fixed to $G=1900 \mathrm{~K} \mathrm{~m}^{-1}$. b) The couple angle $\left(\alpha_{1}, \alpha_{2}\right)$ is fixed to $\left(30^{\circ}, 10^{\circ}\right)$. The temperature gradient, $G$, is fixed to $237.5 \mathrm{~K} \mathrm{~m}^{-1}, 475 \mathrm{~K} \mathrm{~m}^{-1}$ and $1900 \mathrm{~K} \mathrm{~m}^{-1}$. 